\title{
Mapping clustered mutations in cancer reveals APOBEC3 mutagenesis of ecDNA
}

https://doi.org/10.1038/s41586-022-04398-6

Received: 27 May 2021

Accepted: 4 January 2022

Published online: 9 February 2022

\section{Open access}

Check for updates

\author{
Erik N. Bergstrom ${ }^{1,2,3}$, Jens Luebeck ${ }^{4,5}$, Mia Petljak ${ }^{6}$, Azhar Khandekar ${ }^{1,2,3,4}$, Mark Barnes ${ }^{1,2,3}$, \\ Tongwu Zhang ${ }^{7}$, Christopher D. Steele ${ }^{8}$, Nischalan Pillay ${ }^{8,9}$, Maria Teresa Landi ${ }^{7}$, \\ Vineet Bafna ${ }^{5,10}$, Paul S. Mischel ${ }^{11,12}$, Reuben S. Harris ${ }^{13,14,15,16}$ \& Ludmil B. Alexandrov ${ }^{1,2,3 凶}$
}

Clustered somatic mutations are common in cancer genomes and previous analyses reveal several types of clustered single-base substitutions, which include doublet-and multi-base substitutions ${ }^{1-5}$, diffuse hypermutation termed omikli ${ }^{6}$, and longer strand-coordinated events termed kataegis ${ }^{3,7-9}$. Here we provide a comprehensive characterization of clustered substitutions and clustered small insertions and deletions (indels) across 2,583 whole-genome-sequenced cancers from 30 types of cancer $^{10}$. Clustered mutations were highly enriched in driver genes and associated with differential gene expression and changes in overall survival. Several distinct mutational processes gave rise to clustered indels, including signatures that were enriched in tobacco smokers and homologous-recombination-deficient cancers. Doublet-base substitutions were caused by at least 12 mutational processes, whereas most multi-base substitutions were generated by either tobacco smoking or exposure to ultraviolet light. Omikli events, which have previously been attributed to APOBEC3 activity $^{6}$, accounted for a large proportion of clustered substitutions; however, only $16.2 \%$ of omikli matched APOBEC 3 patterns. Kataegis was generated by multiple mutational processes, and $76.1 \%$ of all kataegic events exhibited mutational patterns that are associated with the activation-induced deaminase (AID) and APOBEC3 family of deaminases. Co-occurrence of APOBEC 3 kataegis and extrachromosomal DNA (ecDNA), termed kyklonas (Greek for cyclone), was found in $31 \%$ of samples with ecDNA. Multiple distinct kyklonic events were observed on most mutated ecDNA. ecDNA containing known cancer genes exhibited both positive selection and kyklonic hypermutation. Our results reveal the diversity of clustered mutational processes in human cancer and the role of $A P O B E C 3$ in recurrently mutating and fuelling the evolution of ecDNA.
Cancer genomes contain somatic mutations that are imprinted by different mutational processe ${ }^{1,11}$. Most single-base substitutions and small indels are independently scattered across the genome; however, a subset of substitutions and indels tend to cluster ${ }^{12,13}$. This clustering has been attributed to a combination of heterogeneous mutation rates across the genome, biophysical characteristics of exogenous carcinogens, dysregulation of endogenous processes and larger mutational events associated with genome instability-amongst others ${ }^{2,3,6-8,10,13-19}$. Previous analyses of clustered mutations have focused on single-base substitutions and revealed several classes of clustered events, including doublet-and multi-base substitutions ${ }^{1-5}$ (DBSs and MBSs, respectively), diffuse hypermutation (omikli) ${ }^{6}$ and longer events (kataegis) ${ }^{3,7-9}$. Most kataegic events were found to be strand-coordinated, defined as sharing the same strand and reference allele $e^{3,11}$. Previous studies have also revealed nine clustered signatures ${ }^{13}$ and clustered driver substitutions due to APOBEC 3 -associated mutagenesis ${ }^{6}$ or carcinogenic-triggered POLH mutagenesis ${ }^{13}$.

DBSs have been extensively examined, revealing multiple endogenous and exogenous processes that can cause these events, including failure of DNA repair pathways and exposure to environmental mutagens ${ }^{1,3,11}$. By contrast, MBSs have not been comprehensively investigated, presumably owing to their small numbers in cancer genomes. Moreover, only a handful of processes have been associated with omikli and kataegic events, with most processes attributed to the AID and APOBEC3 family

'Department of Cellular and Molecular Medicine, University of California San Diego, La Jolla, CA, USA. ${ }^{2}$ Department of Bioengineering, University of California San Diego, La Jolla, CA, USA. ${ }^{3}$ Moores Cancer Center, University of California San Diego, La Jolla, CA, USA. ${ }^{4}$ Bioinformatics and Systems Biology Graduate Program, University of California San Diego, La Jolla, CA, USA. ${ }^{5}$ Department of Computer Science and Engineering, University of California San Diego, La Jolla, CA, USA. ${ }^{6}$ Broad Institute of MIT and Harvard, Cambridge, MA, USA. ${ }^{7}$ Division of Cancer Epidemiology and Genetics, National Cancer Institute, Bethesda, MD, USA. ${ }^{8}$ Research Department of Pathology, Cancer Institute, University College London, London, UK. ${ }^{9}$ Department of Cellular and Molecular Pathology, Royal National Orthopaedic Hospital NHS Trust, Stanmore, UK. ${ }^{10} \mathrm{Halıc}$ oğlu Data Science Institute, University of California San Diego, La Jolla, CA, USA. ${ }^{11}$ Department of Pathology, Stanford University School of Medicine, Stanford, CA, USA. ${ }^{12}$ ChEM-H, Stanford University, Stanford, CA, USA. ${ }^{13}$ Howard Hughes Medical Institute, University of

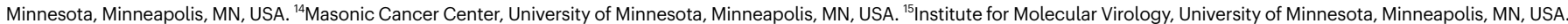
${ }^{16}$ Department of Biochemistry, Molecular Biology and Biophysics, University of Minnesota, Minneapolis, MN, USA. ${ }^{\otimes}$ e-mail: L2alexandrov@health.ucsd.edu 

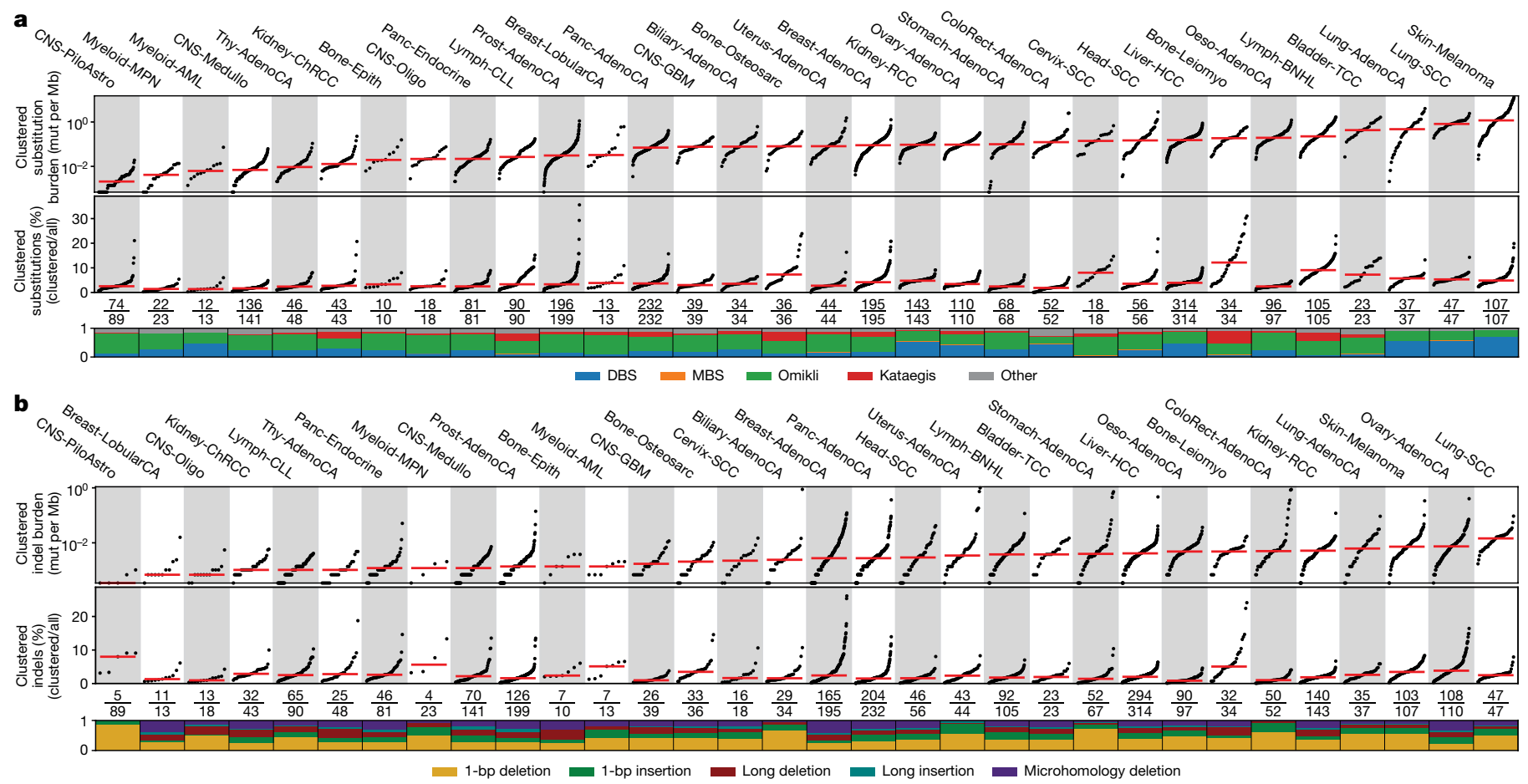

Fig. $1 \mid$ The landscape of clustered mutations across human cancer. a, Pan-cancer distribution of clustered substitutions subclassified into DBSs, MBSs, omikli, kataegis and other clustered mutations. Top, each black dot represents a single cancer genome. Red bars reflect the median clustered TMB (mutations (mut) per Mb) for cancer types. Middle, the clustered TMB normalized to the genome-wide TMB reflecting the contribution of clustered mutations to the overall TMB of a given sample. Red bars reflect the median contribution for cancer types. Bottom, the proportion of each subclass of clustered events for a given cancer type with the total number of samples having at least a single clustered event over the total number of samples within a given cancer cohort. b, Pan-cancer distribution of clustered small indels. The top and middle panels have the same information as a. Bottom, the proportion of each cluster type of indel for a given cancer type with the total number of samples having at least a single clustered indel over the total number of samples within a given cancer cohort. All 2,583 whole-genome-sequenced samples from PCAWG are included in the analysis; however, cancers with fewer than 10 samples were removed from the main figure and included in Extended Data Fig. 1d. For definitions of abbreviations for cancer types used in the figures, see 'Cancer-type abbreviations' in Methods. of deaminases $3,6-8,13,14,20-23$. Specifically, the APOBEC3 enzymes, which are typically responsible for antiviral responses ${ }^{24-30}$, give rise to omikli and kataegis by requiring single-stranded DNA as a substrate $e^{6,8,23,31}$. Omikli were found to be enriched in early replicating regions and more prevalent in microsatellite stable tumours, indicating that mismatch repair has a role in exposing short single-stranded DNA regions ${ }^{6}$. The differential activity of mismatch repair towards gene-rich regions results in increased omikli events within cancer genes ${ }^{6}$. Kataegis is less prevalent than omikli as it is likely to depend on longer tracks of single-stranded $\mathrm{DNA}^{7,8,19}$. Such tracks are typically available during the repair of double-strand breaks and most kataegis has been observed within $10 \mathrm{~kb}$ of detected breakpoints ${ }^{10}$.

Amplification of known cancer genes is known to drive tumorigenesis in many types of cancer ${ }^{32}$. Studies have shown high copy-number states of circular ecDNAs, which often contain known cancer genes and are found in most cancers ${ }^{32-35}$. The circular nature of ecDNAs and their rapid replication mimic double-stranded DNA viral pathogens, which indicates that they could be substrates for APOBEC 3 mutagenesis; this may contribute to the evolution of tumours that contain ecDNA through accelerated diversification of extrachromosomal oncoproteins.

\section{The landscape of clustered mutations}

To identify clustered mutations, a sample-dependent intra-mutational distance (IMD) cut-off was derived in which mutations below the cut-off were unlikely to occur by chance $(q$-value $<0.01)$. A statistical approach using the IMD cut-off, variant allele frequencies (VAFs) and corrections for local sequence context was applied to each specimen (Methods, Extended Data Fig. 1a). Clustered mutations with consistent VAFs were subclassified into four categories (Extended Data Fig. 1b). DBSs and MBSs were characterized as two adjacent mutations (DBSs) and as three or more adjacent mutations (MBSs) (IMD =1). Multiple substitutions each with IMD > 1 bp and below the sample-dependent cut-off were characterized as either omikli (two to three substitutions) or kataegis (four or more substitutions) (Supplementary Fig. 1). Clustered substitutions with inconsistent VAFs were classified as 'other'. Although clustered indels were not subclassified into different categories, most events resembled diffuse hypermutation, with $92.3 \%$ of events having only two indels (Extended Data Fig. 1c).

Examining 2,583 whole-genome-sequenced cancers from the Pan-Cancer Analysis of Whole Genomes (PCAWG) project revealed a total of 1,686,013 clustered single-base substitutions and 21,368 clustered indels (Fig. 1, Extended Data Fig. 1d). DBSs, MBSs, omikli and kataegis comprised $45.7 \%, 0.7 \%, 37.2 \%$ and $7.0 \%$ of clustered substitutions across all samples, respectively, and their distributions varied greatly within and across cancer types. For example, melanoma had the highest clustered substitution burden, with ultraviolet light associated doublets ( $\mathrm{CC}>\mathrm{TT}$ ) accounting for $74.2 \%$ of clustered mutations; however, these contributed only $5.3 \%$ of all substitutions in melanoma (Fig. 1a). By contrast, $11.5 \%$ of all substitutions in bone leiomyosarcomas were clustered, and omikli and kataegis constituted $43.8 \%$ and $46.7 \%$ of these mutations, respectively (Fig. 1a). Clustered indels exhibited similarly diverse patterns within and across cancer types (Fig. 1b). For example, the highest mutational burden of clustered indels was observed in lung and ovarian cancers. Clustered indels in lung cancer accounted for only $2.6 \%$ of all indels and were characterized by 1 -bp deletions. By contrast, clustered long indels at microhomologies were commonly found in 


\section{Article}

ovarian and breast cancers and contributed more than $10 \%$ of all indels in a subset of samples (Fig. 1b). Correlations between the total number of mutations and the number of clustered mutations were observed for DBSs and omikli but not for MBSs, kataegis or indels (Extended Data Fig. 1e). In most cancers, DBSs and omikli had VAFs consistent with those of non-clustered mutations, whereas MBSs and kataegis tended to have lower VAFs (Extended Data Fig. 1f). Kataegic events contained 4 to 44 mutations and $81 \%$ of events were strand-coordinated, indicative of damage or enzymatic changes on a single DNA strand.

The overall survival was compared between patients with cancers containing high and low numbers of clustered mutations within whole-genome-sequenced PCAWG and whole-exome sequenced The Cancer Genome Atlas (TCGA) cancer types ${ }^{36}$. Better overall survival was observed only in whole-genome-sequenced ovarian cancers that contained high-levels of clustered substitutions or clustered indels $(q$-values $<0.05)$ (Extended Data Fig. 1g, h). Conversely, whole-exome-sequenced adrenocortical carcinomas containing clustered substitutions were associated with a worse overall survival $\left(q\right.$-value $\left.=7.2 \times 10^{-5}\right)($ Extended Data Fig. 1i-k).

\section{Signatures of clustered mutations}

Mutational signature analysis was performed for each category of clustered events, which enabled the identification of 12 DBS, 5 MBS, 17 omikli, 9 kataegic and 6 clustered indel signatures (Fig. 2, Supplementary Tables 1-5). Although DBS signatures have previously been described ${ }^{1}$, previous analysis combined DBSs and MBSs into a single class ${ }^{1}$. Separating these events into individual classes showed that a multitude of processes can give rise to DBSs, whereas most MBSs are attributable to signatures associated with tobacco smoking (SBS4) or ultraviolet light (SBS7). Additional DBS and MBS signatures were found within a small subset of cancer types (Extended Data Fig. 2).

In cancer genomes, omikli were previously attributed to APOBEC3 mutagenesis ${ }^{6}$ with some indirect evidence from experimental models $^{23,37,38}$. Our analysis of sequencing data ${ }^{39}$ from the clonally expanded breast cancer cell line BT- 474 with active APOBEC 3 mutagenesis experimentally confirmed the existence of APOBEC3-associated omikli events (cosine similarity: 0.99) (Extended Data Fig. 3a). Only $16.2 \%$ of omikli events across the 2,583 cancer genomes matched the APOBEC 3 mutational pattern, suggesting that a variety of other processes can give rise to diffuse clustered hypermutation. Notably, our analysis revealed omikli due to tobacco smoking (SBS4), clock-like mutational processes (SBS5), ultraviolet light (SBS7), both direct and indirect mutations from AID (SBS9 and SBS85), and multiple mutational signatures with unknown aetiology in different cancer types (SBS8, SBS12, SBS17a/b, SBS28, SBS40 and SBS41) (Fig. 2). Cell lines previously exposed to benzo $[a]$ pyrene ${ }^{40}$ and ultraviolet light ${ }^{41}$ confirmed the generation of omikli events as a result of these two environmental exposures (cosine similarities: 0.86 and 0.84 , respectively) (Extended Data Fig. 3a).

Of the nine kataegic signatures, four have been reported previously, including two associated with APOBEC3 deaminases (SBS2 and SBS13) and two associated with canonical or non-canonical AID activities (SBS84 and SBS85) (Fig. 2). SBS5 (clock-like mutagenesis) accounted for $15.0 \%$ of kataegis, with most events occurring in the vicinity of AID kataegis within B cell lymphomas. The remaining four kataegic signatures accounted for only $8.9 \%$ of kataegic mutations and included SBS7a/b (ultraviolet light), SBS9 (indirect mutations from AID) and SBS37 (unknown aetiology). Most kataegic signatures were strand-coordinated (Extended Data Fig. 3b). Some samples exhibited consistent whereas others exhibited distinct signatures of clustered and non-clustered mutagenesis (Extended Data Fig. 4). For example, in SP56533 (lung squamous cell carcinoma), most non-clustered and omikli substitutions were caused by tobacco signature SBS4, whereas kataegic events were generated by the APOBEC3 signatures (Extended Data Fig. 4a). By contrast, the pattern of non-clustered substitutions in SP24815 (glioblastoma) was due to clock-like signatures SBS1 and

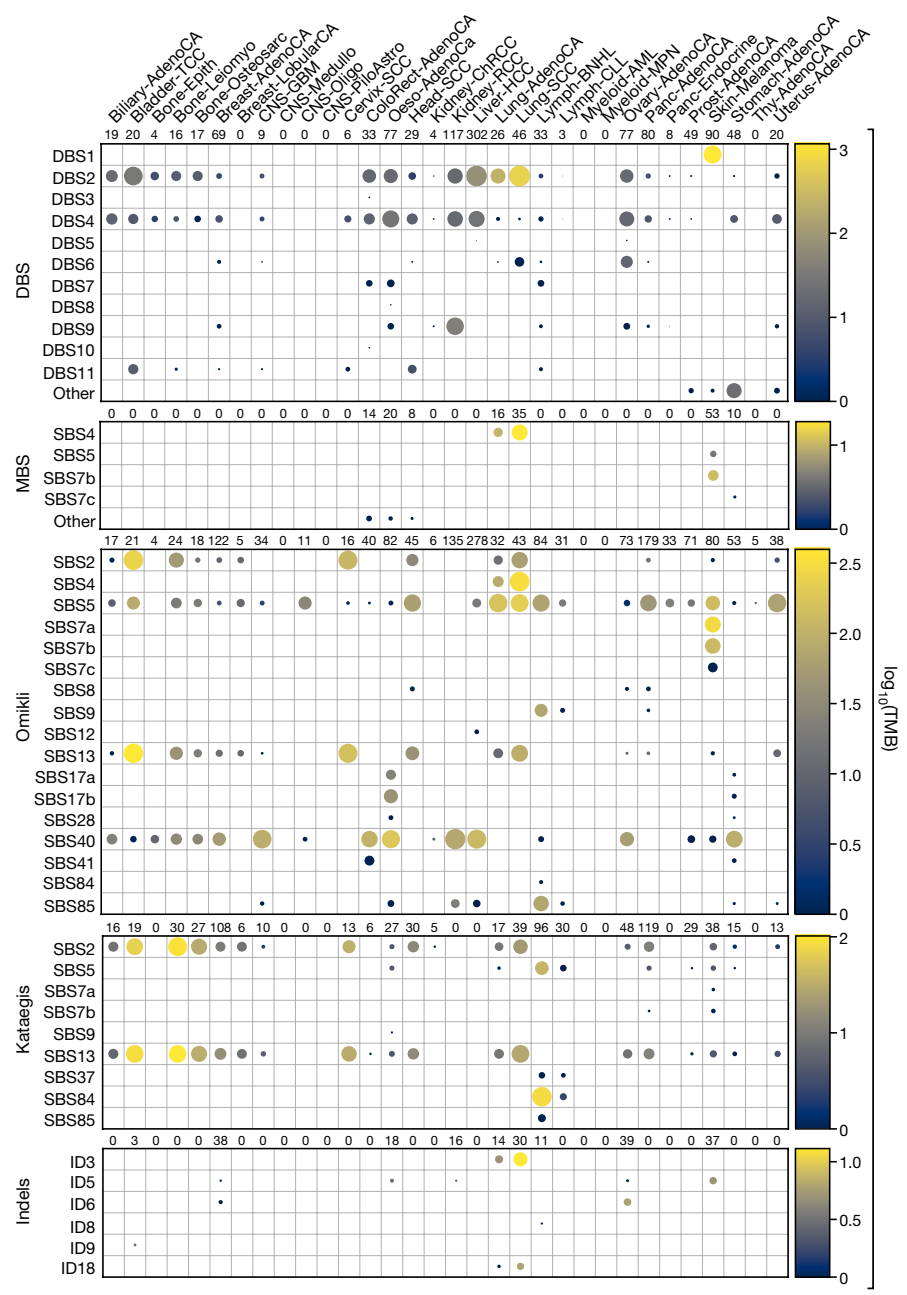

Fig. 2 | Mutational processes that underlie clustered events. Each circle represents the activity of a signature for a given cancer type. The radius of the circle determines the proportion of samples with greater than a given number of mutations specific to each subclass; the colour reflects the median number of mutations per cancer type. A minimum of two samples are required per cancer type for visualization (Methods).

SBS5, whereas omikli and kataegic events were mostly attributable to APOBEC3 (Extended Data Fig. 4a).

The remaining 'other' clustered substitutions exhibited inconsistent VAFs that probably represent mutations at highly mutable genomic regions or the effects of co-occurring large mutational events such as copy number alterations (Extended Data Fig.3d, Supplementary Table 6).

Different cancers showed distinct tendencies of clustered indel mutagenesis (Fig. 2). For instance, clustered indels attributed to ID3 (tobacco smoking; characterized by 1-bp deletions) were found predominately in lung cancers and were significantly increased in smokers compared to non-smokers $(P=0.0014)$ (Extended Data Figs. 3c, $4 \mathrm{~b})$. Clustered indels due to signatures ID6 and ID8-both attributed to homologous recombination deficiency and characterized by long indels at microhomologies-were found in breast and ovarian cancers and were highly increased in cancers with known deficiencies in homologous recombination genes $\left(P=4.9 \times 10^{-11}\right)$ (Extended Data Figs. $\left.3 \mathrm{c}, 4 \mathrm{~b}\right)$.

\section{Panorama of clustered driver mutations}

The PCAWG project elucidated a constellation of mutations that putatively drive cancer development ${ }^{10}$. Our current analysis reveals significant enrichments of clustered substitutions and clustered indels amongst these driver mutations. Specifically, whereas only $3.7 \%$ of 

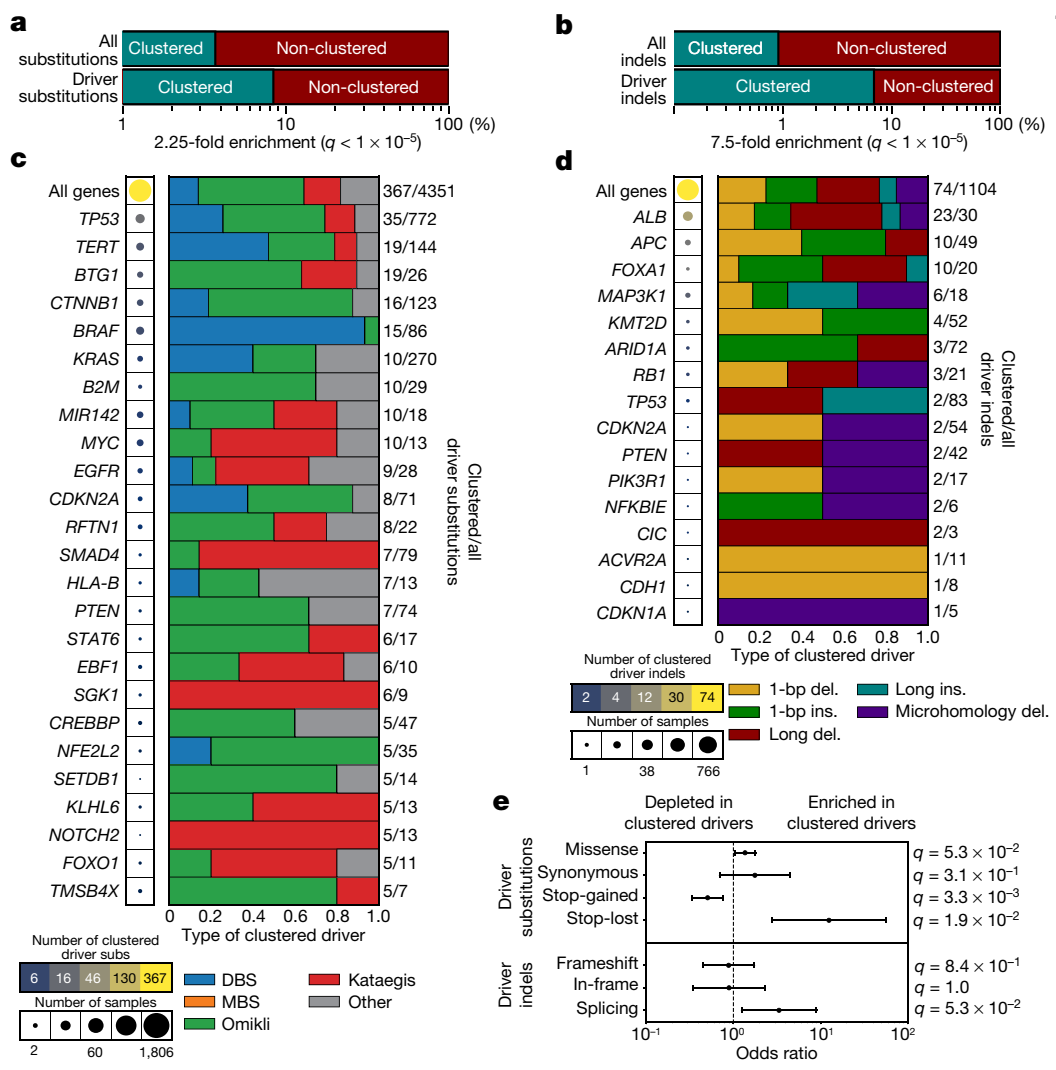
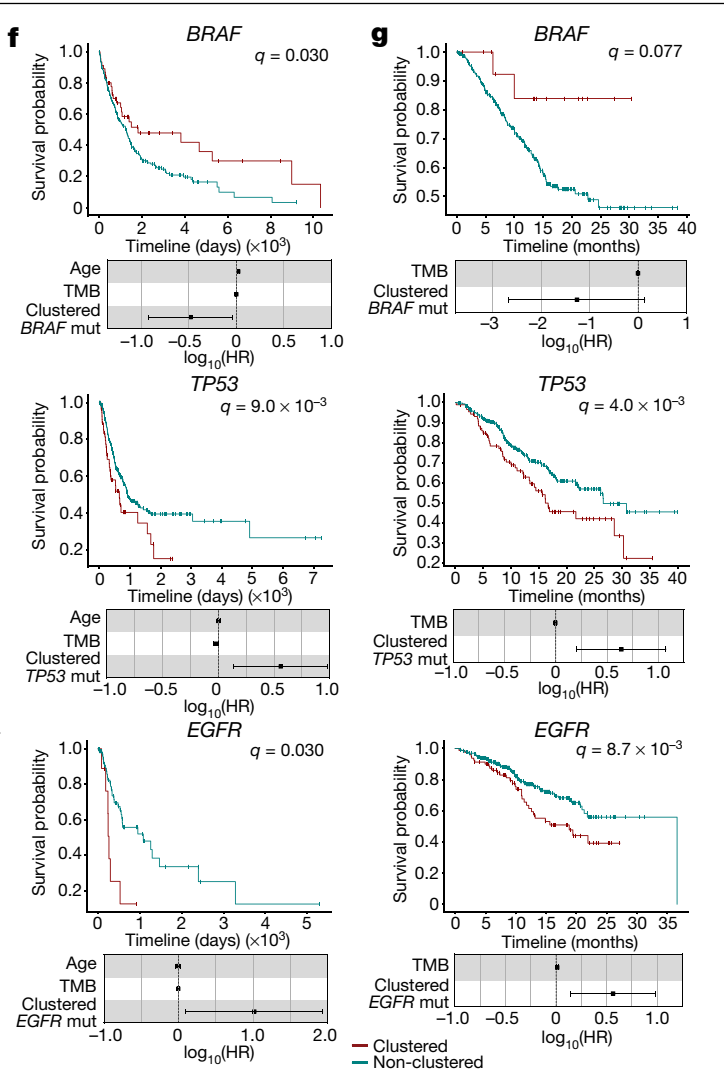

Fig. 3 | Panorama of clustered driver mutations in human cancer.

$\mathbf{a}, \mathbf{b}$, Percentage of clustered mutations (top) compared to the percentage of clustered driver events (bottom) for substitutions (a) and indels (b). c, The frequency of clustered driver events across known cancer genes. The radius of the circle is proportional to the number of samples with a clustered driver mutation within a gene; the colour reflects the clustered mutational burden. All clustered driver events are classified into one of the five clustered classes, with the number of clustered driver substitutions and the total number of driver substitutions shown on the right. d, Clustered indel drivers are shown in a similar manner to c.e, The odds ratio of clustered substitutions (top) and indels (bottom) resulting in deleterious ( $n=192$ clustered substitutions; $n=54$ clustered indels) or synonymous changes ( $n=5$ clustered substitutions; $n=5$ clustered indels) within a given driver gene compared to non-clustered driver mutations ( $n=771$ deleterious and $n=237$ synonymous substitutions; $n=111$ deleterious and $n=50$ synonymous indels). All events were overlapped with the PCAWG consensus list of driver events and were annotated using the ENSEMBL Variant Effect Predictor (VEP). The odds ratios are shown with their $95 \%$ confidence intervals.f, Kaplan-Meier survival curves comparing the outcome of samples with clustered versus non-clustered mutations in BRAF(top), TP53 (middle) and EGFR (bottom) across TCGA cohorts. Only cohorts with more than five samples containing a clustered mutation within the given gene were included.g, Kaplan-Meier survival curves comparing the outcome of samples with clustered versus non-clustered mutations in the same genes across the MSK-IMPACT cohort. The $\log _{10}$-transformed hazards ratios $\left(\log _{10}(\mathrm{HR})\right)$ are shown with their $95 \%$ confidence intervals in $\mathbf{f}, \mathbf{g}$. Cox regressions were corrected for age (TCGA only), mutational burden and cancer type (Methods). $Q$ values in a,b, e were calculated using a two-tailed Fisher's exact test and corrected for multiple hypothesis testing. all substitutions and $0.9 \%$ of all indels are clustered events, they contribute $8.4 \%$ and $6.9 \%$ of substitution and indel drivers, respectively ( $q$-values $<1 \times 10^{-5}$; Fisher's exact tests) (Fig. 3a, b). Omikli accounted for $50.5 \%$ of all clustered substitution drivers, whereas DBSs, kataegis and other clustered events each contributed between $14 \%$ and $18 \%$ (Fig. 3c). Clustered driver substitutions varied greatly between genes and across different cancers (Fig. 3c, Extended Data Fig. 5a) with a 2.4-fold enrichment of clustered events within oncogenes compared to tumour suppressors $\left(P=5.79 \times 10^{-3}\right)$ (Extended Data Fig. 5b, c). In some cancer genes, only a small percentage of driver events are due to clustered substitutions; examples include TP53 (4.5\% clustered driver substitutions), KRAS (3.7\%) and PIK3CA (2.2\%). In other genes, most detected substitution drivers were clustered events; examples include: BTG1 (73.1\%), SGK1 (66.6\%), EBF1 (60.0\%) and NOTCH2 (38.5\%). Notably, the contribution from each class of clustered events varied across driver substitutions in different genes (Fig. 3c). For instance, ultraviolet-light-associated DBSs comprised $93 \%$ of clustered $B R A F$ driver events, omikli contributed $63 \%$ of clustered $B T G 1$ driver events and kataegis accounted for $100 \%$ of clustered $\mathrm{NOTCH} 2$ driver substitutions (Fig. 3c). Similar behaviour was observed for clustered indel drivers, with $48.7 \%$ being single-base pair indels (Fig. $3 d$ ). In some cancer genes, clustered indel drivers were rare (for example, $2.4 \%$ of indel drivers in TP53 were clustered), whereas in others they were common (for example, $76.6 \%$ in $A L B$ ) (Fig. 3d). Clustered driver substitutions were enriched in stop-lost mutations $\left(q\right.$-value $\left.=1.9 \times 10^{-2}\right)$ and depleted in stop-gained mutations $\left(q\right.$-value $\left.=3.3 \times 10^{-3}\right)$ when compared to non-clustered drivers (Fig. 3e). Furthermore, driver genes that contained clustered events were often differentially expressed compared to those containing non-clustered events (Extended Data Fig. 5d). For instance, clustered events within CTNNB1 and BTG1 associated with an increased expression compared to both non-clustered and wild-type expression levels for each gene $(q$-values $<0.05)$. Opposite effects were observed in STAT6 and RFTN1 ( $q$-values $<0.05)$. Collectively, these driver events were induced by the activity of multiple mutational processes including exposure to ultraviolet light, tobacco smoke, platinum chemotherapy and AID and APOBEC 3 activity; amongst others (Extended Data Fig. 5e).

The clinical utility of detecting clustered events in driver genes was evaluated by comparing the survival amongst individuals with clustered mutations versus individuals with non-clustered mutations within 


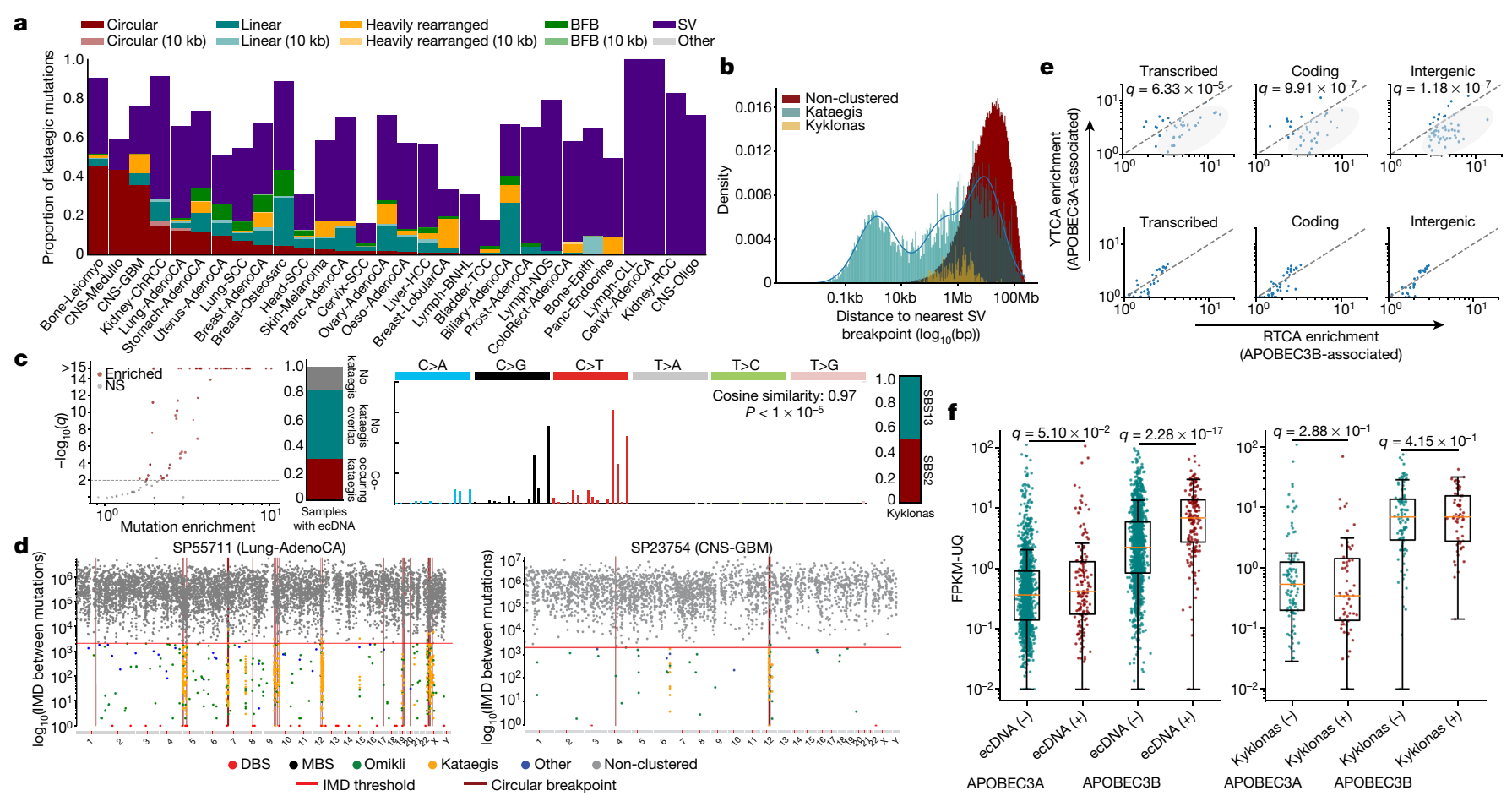

Fig. 4 | Kataegic events co-locate with most forms of structural variation. a, Proportion of all kataegic events per cancer type overlapping different amplifications or structural variations. b, Distance to the nearest breakpoint for all kataegic mutations (teal), kyklonas (gold) and non-clustered mutations (red). Kataegic distances were modelled as a Gaussian mixture with three components (blue line). c, Left, volcano plot depicting samples that are statistically enriched for kyklonas (red; $q$-values from a false discovery rate (FDR)-corrected $z$-test; not significant (NS)). Middle left, proportion of samples with ecDNA co-occurring with kataegis. Middle right, mutational spectrum of all kyklonas. Right, proportion of kyklonic events attributed to SBS2 and SBS13. Cosine similarity was calculated between the kyklonic and the reconstructed spectra composed using SBS2 and SBS13 ( $P$ value from a $Z$-score test).

d, Rainfall plots illustrating the IMD distribution for a given sample with the genomic locations of ecDNA breakpoints (maroon). e, Top, YTㄷA versus RTㄷA enrichments per sample with kyklonas, in which YTㅡA or RTㅡA enrichment is suggestive of higher APOBEC3A or APOBEC3B activity, respectively. Genic mutations were divided into transcribed (template strand) and coding mutations. The RTCA/YTĆA fold enrichments were compared to those of non-clustered mutations (bottom). f, Relative expression of APOBEC3A and APOBEC3B in samples containing ecDNA $(n=157)$ compared to samples without ecDNA $(n=1,364)$ (left), and in samples with ecDNA that have kyklonas $(n=59)$ compared to samples without kyklonas $(n=98)$ (right). Expression values were normalized using fragments per kilobase of exon per million mapped fragment (FPKM) and upper quartile (UQ) normalization obtained from the PCAWG release. $Q$ values in e, f were calculated using a two-tailed Mann-Whitney $U$-test and FDR corrected using the Benjamini-Hochberg procedure. For box plots, the middle line reflects the median, the lower and upper bounds of the box correspond to the first and third quartiles, and the lower and upper whiskers extend from the box by $1.5 \times$ the interquartile range. each driver gene across all whole-exome-sequenced samples in TCGA. For each of these comparisons, we performed Cox regressions considering the effects from age and tumour mutational burden (TMB) while correcting for cancer type and multiple hypothesis testing. These results were validated in targeted panel sequencing data from the Memorial Sloan Kettering-Integrated Mutation Profiling of Actionable Cancer Targets (MSK-IMPACT) cohort $^{42,43}$. These analyses revealed a significant difference in survival between individuals with clustered and individuals with non-clustered mutations detected in TP53, EGFR and BRAF. Specifically, individuals with clustered events within $B R A F$ had a better overall survival compared to individuals with non-clustered events ( $q$-values $<0.05$ ) (Fig. 3f, g). Conversely, in both TCGA and MSK-IMPACT, individuals with clustered mutations in TP53 or EGFR exhibited a significantly worse outcome compared to individuals with non-clustered mutations in each of these genes ( $q$-values < 0.05) (Fig. 3f, g).

\section{Kataegic events and focal amplifications}

In each sample, kataegic mutations were separated into distinct events on the basis of consistent VAFs across adjacent mutations and IMD distances greater than the sample-dependent IMD threshold (Methods). Our analysis revealed that $36.2 \%$ of all kataegic events occurred within $10 \mathrm{~kb}$ of a structural breakpoint but not on detected focal amplifications (Fig. 4a). In addition, $21.8 \%$ of all kataegic events occurred either on a detected focal amplification or within $10 \mathrm{~kb}$ of a focal amplification's structural breakpoints: $9.6 \%$ on circular ecDNA, $6.3 \%$ on linear rearrangements, $3.3 \%$ within heavily rearranged events and $2.6 \%$ associated with breakage-fusion-bridge cycles (BFBs) (Fig. 4a). Finally, $42.0 \%$ of kataegic events were neither within $10 \mathrm{~kb}$ of a structural breakpoint nor on a detected focal amplification. Modelling the distribution of the distances between kataegic events and the nearest structural variations revealed a multi-modal distribution with three components (Fig. 4b): kataegis within $10 \mathrm{~kb}$, around $1 \mathrm{Mb}$, or more than $1.5 \mathrm{Mb}$ of a detected breakpoint. Of note, ecDNA-associated kataegis-termed kyklonas (Greek for cyclone) - had an average distance from the nearest breakpoint of around $750 \mathrm{~kb}$, with only $0.35 \%$ of kyklonic events occurring both on ecDNA and within $10 \mathrm{~kb}$ of a breakpoint (Fig. 4b). These results indicate that kyklonic events are not likely to have occurred because of structural rearrangements during the formation of ecDNA. In most cancer types, DBSs, MBSs, omikli and other cluster events were not found in the vicinity of structural variations (Extended Data Fig. 6a, b).

\section{Recurrent kyklonic mutagenesis of ecDNA}

Although only $9.6 \%$ of kataegic events occur within ecDNA regions, more than $30 \%$ of ecDNAs had one or more associated kyklonic events (Fig. 4c). The mutations within these ecDNA regions were dominated by the APOBEC 3 patterns, which are characterized by strand-coordinated 

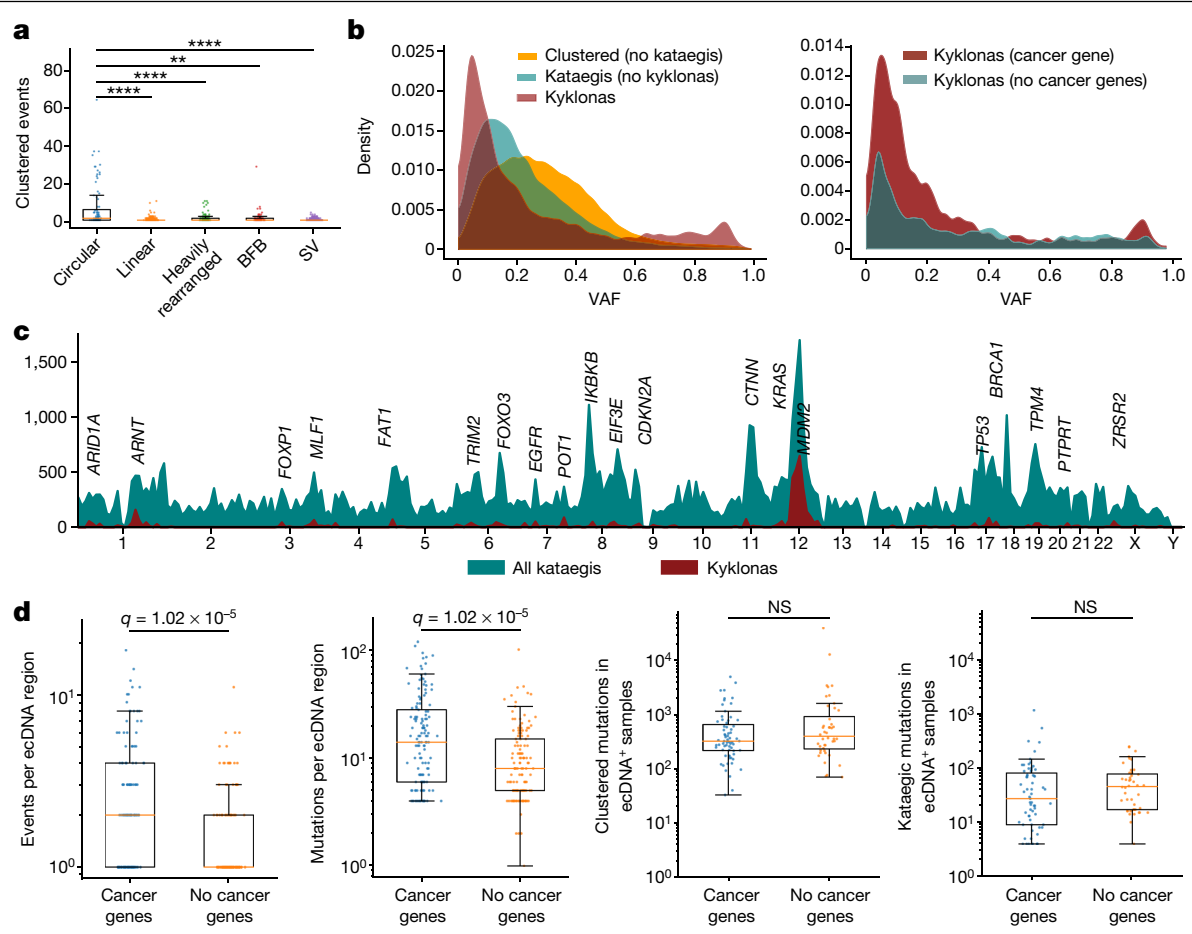

Fig. 5 | Recurrent APOBEC3 hypermutation of ecDNA. a, Number of clustered events overlapping a single amplicon or SV event; each dot represents an amplicon or SV $(n=84$ circular; $n=275$ linear; $n=111$ heavily rearranged; $n=62 \mathrm{BFB}$; and $n=11,139 \mathrm{SV}$ ). A $10-\mathrm{kb}$ window was used to determine the co-occurrence of kataegis with SV breakpoints $\left({ }^{* *} q<0.01\right.$, $\left.{ }^{* * * *} q<0.0001\right)$. b, Left, normalized distributions of the VAFs for all clustered mutations excluding kataegis (orange), all non-ecDNA kataegis (teal), and kyklonas (red). Right, normalized VAF distributions for kyklonic ecDNA containing cancer genes and for kyklonic ecDNA without cancer genes.c,

$\mathrm{C}>\mathrm{G}$ and $\mathrm{C}>\mathrm{T}$ mutations in the Tp$\underline{\mathrm{CpW}}$ context and attributed to signatures SBS2 and SBS13 $\left(P<1 \times 10^{-5}\right)$ (Fig $4 \mathrm{c}$, d, Extended Data Fig. 6c). These APOBEC3-associated events contributed $97.8 \%$ of all kyklonic events, whereas the remaining mutations were attributed to clock-like signature SBS5 (1.2\%) and other signatures (1.0\%) (Extended Data Fig. 6c). Furthermore, kyklonic events exhibited an enrichment of $\mathrm{C}>\mathrm{T}$ and $\mathrm{C}>\mathrm{G}$ mutations at APOBEC3B-preferred RTCA compared to APOBEC3A-preferred YTCA contexts (underlining reflects the mutated nucleotide) ${ }^{7}$, indicating that APOBEC3B is likely to have an important role in the mutagenesis of circular DNA bodies (Fig. 4e). Similar levels of enrichment for RTCA contexts were also observed in both non-ecDNA kataegis and non-structural variant (SV)-associated kataegis, suggesting that APOBEC3B generally gives rise to many of the strand-coordinated kataegic events (Extended Data Fig. 6d). An increase in the expression of APOBEC3B-but not APOBEC3Awas observed in cancers with ecDNA compared to samples without ecDNA (3.1-fold; $q$-value $<1 \times 10^{-5}$ ) (Fig. 4 f). Within cancers containing ecDNA, no differences were observed in the expression of APOBEC3A or APOBEC3B between samples with and without kyklonic events (Fig. 4f).

More recurrent APOBEC 3 kataegis was observed across circular ecDNA regions compared to other forms of structural variation (Fig. 5a). An average of $2.5 \mathrm{kyklonic}$ events were observed within ecDNA regions (range: 0-64 kyklonic events; 0-505 mutations). Recurrent kyklonas was widespread across cancer types (Extended Data Fig. 7a, b). For example, glioblastomas and sarcomas exhibited an average of 5 and 86 kyklonic mutations, respectively. The average VAF of kyklonas was significantly lower than both non-ecDNA associated kataegis and all other clustered events ( $q$-values $\left.<1 \times 10^{-5} \mathrm{Fig} .5 \mathrm{~b}\right)$. Notably, a subset of kyklonas exhibited VAFs above 0.80 , which is likely to reflect early

Frequency of recurrence for all kataegis (teal) and kyklonas (red) using a sliding genomic window of $10 \mathrm{Mb}$. d, Number of kyklonic events and kyklonic mutations per ecDNA region containing cancer genes $(n=137)$ or without cancer genes ( $n=134$; left and right, respectively). e, Total number of clustered and kataegic mutations found in samples with ecDNAs containing cancer genes ( $n=67$ samples) compared to samples with ecDNAs without cancer genes ( $n=44$; left and right, respectively). $Q$ values in a, d, e were calculated using a two-tailed Mann-Whitney $U$-test and FDR-corrected using the BenjaminiHochberg procedure. Box plot parameters as in Fig. 4.

mutagenesis of genomic regions that have subsequently amplified as ecDNA. Moreover, kyklonic events with high VAFs occurred more commonly on ecDNA that contained known cancer genes, suggesting a mechanism of positive selection (Fig. 5b). Approximately 7.2\% of kyklonas occurred early in the evolution of a given ecDNA population within a tumour $(\mathrm{VAF}>0.80)$, whereas the majority of kyklonic events (around $82.5 \% ; \mathrm{VAF}<0.5$ ) have probably occurred after clonal amplification by recurrent $\mathrm{APOBEC} 3$ mutagenesis.

Recurrent kyklonic events were increased within or near known cancer-associated genes including TP53, $C D K 4$ and $M D M 2$, amongst others (Fig. 5c). These recurrent kyklonas were observed across many cancers including glioblastomas, sarcomas, head and neck carcinomas and lung adenocarcinomas (Extended Data Fig. 7c, d). For example, in a sarcoma sample (SP121828), 10 distinct kyklonic events overlapped a single ecDNA region with recurrent APOBEC 3 activity in proximity to $M D M 2$, resulting in a missense $\mathrm{L} 230 \mathrm{~F}$ mutation (Extended Data Fig.7c). The same ecDNA region contained additional kyklonic events occurring within intergenic regions that have distinguishable VAF distributions, implicating recurrent mutagenesis (Extended Data Fig. 7c). Similarly, two distinct kyklonic events occurred on an ecDNA containing EGFR, resulting in a missense mutation D191N within a head and neck cancer (Extended Data Fig. 7d). Of note, ecDNA regions with known cancer-associated genes had significantly higher numbers of kyklonic events and mutational burdens of kyklonas compared to ecDNA regions without any known cancer-associated genes $\left(q\right.$-values $\left.<1 \times 10^{-5}\right)$ (Fig. 5d). Furthermore, we observed a higher co-occurrence of kyklonas with known cancer-associated genes, which were mutated 2.5 times more than ecDNA without cancer-associated genes $\left(P=1.2 \times 10^{-5}\right.$; Fisher's exact test). Overall, $41 \%$ of kyklonic events were found within 
the footprints of known cancer driver genes $\left(P<1 \times 10^{-5}\right)$. These enrichments cannot be accounted for either by an increase in the overall mutations or by an increase in the overall clustered mutations in these samples (Fig. 5e). To understand the functional effect of kyklonas, we annotated the predicted consequence of each mutation. In total, 2,247 kyklonic mutations overlapped putative cancer-associated genes, of which $4.3 \%$ occur within coding regions (Extended Data Fig. 7e). Specifically, 63 resulted in missense mutations, 29 resulted in synonymous mutations, 4 introduced premature stop codons and 1 removed a stop codon (Supplementary Table 7). These downstream consequences of APOBEC 3 mutagenesis suggest a contribution to the oncogenic evolution of specific ecDNA populations.

\section{Validation of kyklonic events in ecDNA}

Kyklonic events were further investigated across 3 additional independent cohorts, including 61 sarcomas ${ }^{44}, 280$ lung cancers ${ }^{45}$ and 186 oesophageal squamous cell carcinomas ${ }^{46}$. Comparable rates of clustered mutagenesis were found for both substitutions and indels to the rates reported in PCAWG, with a 2.4- and 5.0-fold enrichment of clustered substitutions and indels within driver events, respectively (Extended Data Fig. 8a). Across the three cohorts, 31\% of samples with ecDNA exhibited kyklonas within the sarcomas, $14 \%$ within the oesophageal cancers and $28 \%$ within the lung cancers, supporting the rates observed in PCAWG (Fig. 4c, Extended Data Figs. 7b, 8c). Similar to the rate observed in PCAWG (36.2\%), approximately $30.1 \%$ of all kataegis occurred within $10 \mathrm{~kb}$ of the nearest breakpoint in the validation cohort (Extended Data Fig. 9a). In addition, only $0.34 \%$ of kyklonic events in the validation dataset occurred closer to SVs than expected by chance, which closely resembles the observations in the PCAWG data $(0.35 \%)$ (Extended Data Fig. 9b). Kyklonic mutations were predominantly attributed to APOBEC3 signatures SBS2 and SBS13 $\left(P<1 \times 10^{-5}\right)$ (Extended Data Fig. 8b, Methods) with an enrichment of mutations at the RTCA context supporting the role of APOBEC3B (Extended Data Fig. 8d). A widespread recurrence of kyklonic events was observed across the sarcomas, oesophageal and lung cancers, with $45 \%, 28 \%$ and $46 \%$ of samples with ecDNA containing multiple, distinct kyklonic events (Extended Data Fig. 8e). An example from each cohort was selected to illustrate multiple kyklonic events occurring within single ecDNAs, validating the recurrent APOBEC3 hypermutation of ecDNA (Extended Data Fig. 10).

\section{Discussion}

Clustered mutagenesis in cancer can occur through different mutational processes, with AID and APOBEC3 deaminases having the most prominent role. In addition to enzymatic deamination, other endogenous and exogenous sources imprint many of the observed clustered indels and substitutions. A multitude of mutational processes can give rise to omikli events, including tobacco carcinogens and exposure to ultraviolet light. Clustered substitutions and indels were highly enriched in driver events and associated with differential gene expression, implicating them in cancer development and cancer evolution. Some clustered mutational signatures are associated with known cancer risk factors or the activity or failure of DNA repair processes. Notably, clustered mutations in TP53, EGFR and BRAF associated with changes in overall survival and can be detected in most types of sequencing data, including clinically actionable targeted panels such as MSK-IMPACT.

A large proportion of kataegic events occur within $10 \mathrm{~kb}$ of detected SV breakpoints with a mutational pattern, suggesting the activity of APOBEC3. Multiple distinct kataegic events, independent of detected breakpoints, were observed on circular ecDNA; such events-termed kyklonas-suggest recurrent APOBEC3 mutagenesis. The circular topology of ecDNAs ${ }^{47}$ and their rapid replication patterns are reminiscent of the structure and behaviour of the circular genomes of several double-stranded-DNA based, pathogens including herpesviruses, papillomaviruses and polyomaviruses ${ }^{32-35}$. Previous pan-virome studies have shown that these double-stranded DNA viral genomes often manifest mutations from APOBEC 3 enzymes $^{48-50}$. As such, recurrent APOBEC3 mutagenesis on ecDNA is likely to be representative of an antiviral response in which the ecDNA viral-like structure is treated as an infectious agent and attacked by APOBEC 3 enzymes. ecDNAs contain a plethora of cancer-associated genes and are responsible for many gene amplification events that can accelerate tumour evolution. Repeated mutagenic attacks of these ecDNAs reveal functional effects within known oncogenes and implicate additional modes of oncogenesis that may ultimately contribute to subclonal tumour evolution, subsequent evasion of therapy and clinical outcome. Further investigations with large-scale clinically annotated whole-genome-sequenced cancers are required to fully understand the clinical implications of clustered mutations and kyklonas.

\section{Online content}

Any methods, additional references, Nature Research reporting summaries, source data, extended data, supplementary information, acknowledgements, peer review information; details of author contributions and competing interests; and statements of data and code availability are available at https://doi.org/10.1038/s41586-022-04398-6.

1. Alexandrov, L. B. et al. The repertoire of mutational signatures in human cancer. Nature 578, 94-101 (2020).

2. Matsuda, T., Kawanishi, M. Yagi, T. Matsui, S. \& Takebe, H. Specific tandem GG to TT base substitutions induced by acetaldehyde are due to intra-strand crosslinks between adjacent guanine bases. Nucleic Acids Res. 26, 1769-1774 (1998).

3. Nik-Zainal, S. et al. Mutational processes molding the genomes of 21 breast cancers. Cell 149, 979-993 (2012)

4. de Gruijl, F. R., van Kranen, H. J. \& Mullenders, L. H. UV-induced DNA damage, repair, mutations and oncogenic pathways in skin cancer. J. Photochem. Photobiol. B 63, 19-27 (2001).

5. Brash, D. E. UV signature mutations. Photochem. Photobiol. 91, 15-26 (2015).

6. Mas-Ponte, D. \& Supek, F. DNA mismatch repair promotes APOBEC3-mediated diffuse hypermutation in human cancers. Nat. Genet. 52, 958-968 (2020).

7. Chan, $K$. et al. An APOBEC3A hypermutation signature is distinguishable from the signature of background mutagenesis by APOBEC3B in human cancers. Nat. Genet. 47, 1067-1072 (2015).

8. Taylor, B. J. et al. DNA deaminases induce break-associated mutation showers with implication of APOBEC3B and 3A in breast cancer kataegis. Elife 2, e00534 (2013).

9. Boichard, A., Tsigelny, I. F. \& Kurzrock, R. High expression of PD-1 ligands is associated with kataegis mutational signature and APOBEC3 alterations. Oncoimmunology 6 , e1284719 (2017)

10. The ICGC/TCGA Pan-Cancer Analysis of Whole Genomes Consortium. Pan-cancer analysis of whole genomes. Nature 578, 82-93 (2020).

11. Alexandrov, L. B. et al. Signatures of mutational processes in human cancer. Nature $\mathbf{5 0 0}$ 415-421 (2013).

12. Lawrence, M. S. et al. Mutational heterogeneity in cancer and the search for new cancer-associated genes. Nature 499, 214-218 (2013).

13. Supek, F. \& Lehner, B. Clustered mutation signatures reveal that error-prone DNA repair targets mutations to active genes. Cell 170, 534-547 (2017).

14. Buisson, R. et al. Passenger hotspot mutations in cancer driven by APOBEC3A and mesoscale genomic features. Science 364, eaaw2872 (2019).

15. Greenman, C., Wooster, R., Futreal, P. A., Stratton, M. R. \& Easton, D. F. Statistical analysis of pathogenicity of somatic mutations in cancer. Genetics 173, 2187-2198 (2006).

16. Martincorena, I. et al. Universal patterns of selection in cancer and somatic tissues. Cell 171, 1029-1041 (2017)

17. Hainaut, P. \& Pfeifer, G. P. Patterns of p53 G->T transversions in lung cancers reflect the primary mutagenic signature of DNA-damage by tobacco smoke. Carcinogenesis $\mathbf{2 2}$ 367-374 (2001).

18. Pfeifer, G. P., You, Y. H. \& Besaratinia, A. Mutations induced by ultraviolet light. Mutat. Res. 571, 19-31 (2005).

19. Roberts, S. A. et al. Clustered mutations in yeast and in human cancers can arise from damaged long single-strand DNA regions. Mol. Cell 46, 424-435 (2012).

20. Kasar, S. et al. Whole-genome sequencing reveals activation-induced cytidine deaminase signatures during indolent chronic lymphocytic leukaemia evolution. Nat. Commun. 6, 8866 (2015).

21. Roberts, S. A. et al. An APOBEC cytidine deaminase mutagenesis pattern is widespread in human cancers. Nat. Genet. 45, 970-976 (2013).

22. Burns, M. B., Temiz, N. A. \& Harris, R. S. Evidence for APOBEC3B mutagenesis in multiple human cancers. Nat. Genet. 45, 977-983 (2013).

23. Petljak, M. et al. The APOBEC3A deaminase drives episodic mutagenesis in cancer cells. Preprint at https://doi.org/10.1101/2021.02.14.431145 (2021)

24. Bogerd, H. P., Wiegand, H. L., Doehle, B. P., Lueders, K. K. \& Cullen, B. R. APOBEC3A and APOBEC3B are potent inhibitors of LTR-retrotransposon function in human cells. Nucleic Acids Res. 34, 89-95 (2006) 
25. Malim, M. H. \& Bieniasz, P. D. HIV restriction factors and mechanisms of evasion. Cold Spring Harb. Perspect. Med. 2, a006940 (2012).

26. Malim, M. H. Natural resistance to HIV infection: the Vif-APOBEC interaction. C. R. Biol. 329, 871-875 (2006).

27. Venkatesan, $S$. et al. Perspective: APOBEC mutagenesis in drug resistance and immune escape in HIV and cancer evolution. Ann. Oncol. 29, 563-572 (2018).

28. Chen, H. et al. APOBEC $3 A$ is a potent inhibitor of adeno-associated virus and retrotransposons. Curr. Biol. 16, 480-485 (2006).

29. Harris, R. S. \& Dudley, J. P. APOBECs and virus restriction. Virology 479-480, 131-145 (2015).

30. Harris, R. S. et al. DNA deamination mediates innate immunity to retroviral infection. Cell 113, 803-809 (2003)

31. Maciejowski, J. et al. APOBEC3-dependent kataegis and TREX1-driven chromothripsis during telomere crisis. Nat. Genet. 52, 884-890 (2020).

32. Turner, K. M. et al. Extrachromosomal oncogene amplification drives tumour evolution and genetic heterogeneity. Nature 543, 122-125 (2017).

33. Koche, R. P. et al. Extrachromosomal circular DNA drives oncogenic genome remodeling in neuroblastoma. Nat. Genet. 52, 29-34 (2020).

34. Kim, H. et al. Extrachromosomal DNA is associated with oncogene amplification and poor outcome across multiple cancers. Nat. Genet. 52, 891-897 (2020).

35. Verhaak, R. G. W., Bafna, V. \& Mischel, P. S. Extrachromosomal oncogene amplification in tumour pathogenesis and evolution. Nat. Rev. Cancer 19, 283-288 (2019).

36. The Cancer Genome Atlas Research Network et al. The Cancer Genome Atlas Pan-Cancer analysis project. Nat. Genet. 45, 1113-1120 (2013).

37. Green, A. M. et al. APOBEC3A damages the cellular genome during DNA replication. Cell Cycle 15, 998-1008 (2016).

38. Stenglein, M. D., Burns, M. B., Li, M., Lengyel, J. \& Harris, R. S. APOBEC3 proteins mediate the clearance of foreign DNA from human cells. Nat. Struct. Mol. Biol. 17, 222-229 (2010).

39. Petljak, M. et al. Characterizing mutational signatures in human cancer cell lines reveals episodic APOBEC mutagenesis. Cell 176, 1282-1294 (2019).

40. Kucab, J. E. et al. A compendium of mutational signatures of environmental agents. Cell 177, 821-836 (2019).

41. Liu, Z. et al. Human tumor p53 mutations are selected for in mouse embryonic fibroblasts harboring a humanized p53 gene. Proc. Natl Acad. Sci. USA 101, 2963-2968 (2004).

42. Cheng, D. T. et al. Memorial Sloan Kettering-Integrated Mutation Profiling of Actionable Cancer Targets (MSK-IMPACT): a hybridization capture-based next-generation sequencing clinical assay for solid tumor molecular oncology. J. Mol. Diagn. 17, 251-264 (2015).

43. Zehir, A. et al. Mutational landscape of metastatic cancer revealed from prospective clinical sequencing of 10,000 patients. Nat. Med. 23, 703-713 (2017).

44. Steele, C. D. et al. Undifferentiated sarcomas develop through distinct evolutionary pathways. Cancer Cell 35, 441-456 (2019).

45. Zhang, T. et al. Genomic and evolutionary classification of lung cancer in never smokers. Nat. Genet. 53, 1348-1359 (2021).

46. Moody, S. et al. Mutational signatures in esophageal squamous cell carcinoma from eight countries with varying incidence. Nat. Genet. 53, 1553-1563 (2021).

47. Wu, S. et al. Circular ecDNA promotes accessible chromatin and high oncogene expression. Nature 575, 699-703 (2019).

48. Cheng, A. Z. et al. Epstein-Barr virus BORF2 inhibits cellular APOBEC3B to preserve viral genome integrity. Nat. Microbiol. 4, 78-88 (2019).

49. Poulain, F., Lejeune, N., Willemart, K. \& Gillet, N. A. Footprint of the host restriction factors APOBEC3 on the genome of human viruses. PLoS Pathog. 16, e1008718 (2020).

50. Zhu, B. et al. Mutations in the HPV16 genome induced by APOBEC3 are associated with viral clearance. Nat. Commun. 11, 886 (2020).

Publisher's note Springer Nature remains neutral with regard to jurisdictional claims in published maps and institutional affiliations.

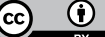
and reproduction in any medium or format, as long a you give appropriate credit to the original author(s) and the source, provide a link to the Creative Commons license and indicate if changes were made. The images or other third party material in this article are included in the article's Creative Commons license, unless indicated otherwise in a credit line to the material. If material is not included in the article's Creative Commons license and your intended use is not permitted by statutory regulation or exceeds the permitted use, you will need to obtain permission directly from the copyright holder. To view a copy of this license, visit http://creativecommons.org/licenses/by/4.0/.

(c) The Author(s) 2022 


\section{Article}

\section{Methods}

\section{Data sources}

Somatic variant calls of single-base substitutions, small indels and structural variations were downloaded for the 2,583 white-listed whole-genome-sequenced samples from PCAWG along with the corresponding list of consensus driver events ${ }^{10}$. Epidemiological and clinical features for all available samples were downloaded from the official PCAWG release (https://dcc.icgc.org/releases/PCAWG). The collection of whole-exome-sequenced samples from TCGA along with all available clinical features were downloaded from the Genomic Data Commons (GDC; https://gdc.cancer.gov/). The MSK-IMPACT Clinical Sequencing Cohort ${ }^{43}$ composed of 10,000 clinical cases was downloaded from cBioPortal (https://www.cbioportal.org/study/summary?id=msk impact_2017). The subclassification of focal amplifications comprised circular ecDNA, linear amplifications, BFBs and heavily rearranged events, and their corresponding genomic locations were obtained for a subset of samples $(n=1,291)$ as reported ${ }^{34}$.

Experimental models used to validate clustered events were derived from previous studies using primary Hupki mouse embryonic fibroblasts (MEFs) exposed to ultraviolet light ${ }^{41}$, human induced pluripotent stem cells (iPS cells) exposed to benzo $[a]$ pyrene ${ }^{40}$, and a clonally expanded BT-474 human breast cancer cell line with episodically active APOBEC $3^{39}$.

Independent cohorts used to validate kyklonic events were collected from multiple sources. The 61 undifferentiated sarcomas ${ }^{44}$ and 187 high-confidence oesophageal squamous cell carcinomas ${ }^{46}$ were downloaded from the European Genome-phenome Archive (EGAD00001004162 and EGAD00001006868, respectively). The 280 lung adenocarcinomas ${ }^{45}$ were downloaded from $\mathrm{dbGaP}$ under the accession number (phs001697.v1.p1). Clustered mutations in validation samples were analysed using the same approach as the one used in the original cohort.

\section{Detection of clustered events}

SigProfilerSimulator (v.1.0.2) was used to derive an IMD cut-off ${ }^{51}$ that is unlikely to occur by chance based on the TMB and the mutational patterns for a given sample. Specifically, each tumour sample was simulated while maintaining the sample's mutational burden on each chromosome, the \pm 2 bp sequence context for each mutation and the transcriptional strand bias ratios across all mutations. All mutations in each sample were simulated 100 times and the IMD cut-off was calculated such that $90 \%$ of the mutations below this cut-off could not appear by chance $(q$-value $<0.01)$. For example, in a sample with an IMD threshold of $500 \mathrm{bp}$, one may observe 1,000 mutations within this threshold with no more than 100 mutations expected based on the simulated data $(q$-value $<0.01)$. $P$ values were calculated using $z$-tests by comparing the number of real mutations and the distribution of simulated mutations that occur below the same IMD threshold. A maximum cut-off of $10 \mathrm{~kb}$ was used for all IMD thresholds. By generating a background distribution that reflects the random distribution of events used to reduce the false positive rate, this model also considers regional heterogeneities of mutation rates, partially attributed to replication timing and expression, and variances in clonality by correcting for mutation-rich regions and mutation-poor regions within 1-Mb windows. The 1-Mb window size has been used and established as an appropriate scale when considering the variability in mutation rates associated with chromatin structure, replication timing and genome architecture ${ }^{14,52,53}$. The 1-Mb window ensures that subsequent mutations are likely to have occurred as single events using a maximum cut-off of 0.10 for differences in the VAFs. The regional IMD cut-off was determined using a sliding window approach that calculated the fold enrichment between the real and simulated mutation densities within 1-Mb windows across the genome. The IMD cut-offs were further increased, for regions that had higher than ninefold enrichments of clustered mutations and where more than $90 \%$ of the clustered mutations were found within the original data, to capture additional clustered events while maintaining the original criteria (less than $10 \%$ of the mutations below this cut-off appear by chance; $q$-value $<0.01$ ). Last, as VAF of mutations may confound the definition of clustered events in ecDNA, we calculated the distribution of inter-event distances within recurrently mutated ecDNA while disregarding the VAF of individual mutations. This resulted in the exact same separation of kataegic events using only the inter-event distances as a criterion for the grouping of mutations into a single event.

Subsequently, all clustered mutations with consistent VAFs were classified into one of four categories (Extended Data Fig. 1a). Two adjacent mutations with an IMD of 1 were classified as DBSs. Three or more adjacent mutations each with an IMD of 1 were classified as MBSs. Two or three mutations with IMDs less than the sample-dependent threshold and with at least a single IMD greater than 1 were classified as omikli. Four or more mutations with IMDs less than the sample-dependent threshold and with at least a single IMD greater than 1 were classified as kataegis. A cut-off of four mutations for kataegis was chosen by fitting a Poisson mixture model to the number of mutations involved in a single event across all extended clustered events excluding DBSs and MBSs (Supplementary Note1). This model comprised two distributions with $\mathrm{C} 1=2.08$ and $\mathrm{C} 2=4.37$ representing omikli and kataegis, respectively. A cut-off of four mutations was used for kataegis on the basis of a contribution of greater than $95 \%$ from the kataegis-associated distribution with events of four or more mutations. Note that there is certain ambiguity for events with two or three mutations. Although the majority of these events are omikli, some of these events are likely to be short kataegic events (Supplementary Note 1 ). All remaining clustered mutations with inconsistent VAFs were classified as other. Clustered indels were not classified into different classes. We also performed additional quality-checks to ensure that the majority of clustered indels were mapped to high confidence regions of the genome (Supplementary Fig. 2). Specifically, all clustered indels were aligned against a consensus list of blacklisted genomic regions developed by $\mathrm{ENCODE}^{54}$ revealing that only $0.5 \%$ of all clustered indels overlapped regions with low mappability scores.

\section{Clustered mutational signatures analysis}

The clustered mutational catalogues of the examined samples were summarized in SBS288 and ID83 matrices using SigProfilerMatrixGenerator $^{55}$ (v.1.2.0) for each tissue type and each category of clustered events. For example, six matrices were constructed for clustered mutations found in Breast-AdenoCA: one matrix for DBSs, one matrix for MBSs, one matrix for omikli, one matrix for kataegis, one matrix for other clustered substitutions and one matrix for clustered indels. The SBS288 classification considers the $5^{\prime}$ and $3^{\prime}$ bases immediately flanking each single-base substitution (referred to using the pyrimidine base in the Watson-Crick base pair) resulting in 96 individual mutation channels. In addition, this classification considers the strand orientation for mutations that occur within genic regions resulting in three possible categories: (1) transcribed; pyrimidine base occurs on the template strand; (2) untranscribed; pyrimidine base occurs on the coding strand; or (3) non-transcribed; pyrimidine base occurs in an intergenic region. Mutations in genic regions that are bidirectionally transcribed were evenly split amongst the coding and template strand channels. Combined, this results in a classification consisting of 288 mutation channels, which were used as input for de novo signature extraction of clustered substitutions. The ID83 mutational classification has previously been described ${ }^{55}$.

Mutational signatures were extracted from the generated matrices using SigProfilerExtractor (v.1.1.0), a Python-based tool that uses non-negative matrix factorization to decipher both the number of operative processes within a given cohort and the relative activities of each process within each sample $e^{56}$. The algorithm was initialized 
using random initialization and by applying multiplicative updates using the Kullback-Leibler divergence with 500 replicates. Each de novo extracted mutational signature was subsequently decomposed into the COSMIC (v.3) set of signatures (https://cancer.sanger. ac.uk/signatures/) requiring a minimum cosine similarity of 0.80 for all reconstructed signatures. All de novo extractions and subsequent decomposition were visually inspected and, as previously done ${ }^{1}$, manual corrections were performed for $2.2 \%$ of extractions ( 4 out of 180 extractions) in which the total number of operative signatures was adjusted \pm 1 . Consistent with prior visualizations ${ }^{10}$, we have included all cancer types within the PCAWG cohort, which may comprise as few as one sample for certain cancer types. Similarly, consistent with prior visualizations ${ }^{1}$, decomposed signature activity plots required that each cancer type have more than 2 samples and used mutation thresholds for each clustered category; 25 mutations per sample were required for DBSs, omikli events and other clustered mutations; 15 mutations per sample were required for MBSs and kataegic events; and 10 mutations were required per sample for clustered indels.

\section{Experimental validation}

A subset of clustered mutational signatures was validated using previously sequenced in vitro cell line models. As done for PCAWG samples, we generated a background model using SigProfilerSimulator ${ }^{51}$ to calculate the clustered IMD cut-off for each sample and partitioned each substitution into the appropriate category of clustered events. Mutational spectra were generated for each subclass within each sample using SigProfilerMatrixGenerator ${ }^{55}$ and were compared against the de novo signatures extracted from human cancer. The cosine similarity between the in vitro mutational spectra and de novo observed clustered signatures was calculated to assess the degree of similarity. The average cosine similarity between two random non-negative vectors is 0.75 , and the cosine similarities above 0.81 reflect $P$ values below 0.01 (ref. ${ }^{51}$ ).

\section{Associations with cancer risk factors}

Homologous recombination (HR) deficiency was defined for breast cancers using the status of $B R C A 1, B R C A 2, R A D 51 C$ and $P A L B 2^{57}$. Samples with a germline, somatic or epigenetic alteration in one of these genes were considered HR-deficient, whereas samples without any known alterations in these genes were considered HR-proficient. The number of clustered indels was compared between HR-deficient and HR-proficient samples. The smoking status of lung cancers was determined using the clinical annotation from TCGA (https://portal.gdc.cancer.gov/repository). The number of clustered indels associated with tobacco smoking (ID6) was compared between samples annotated as lifelong non-smokers and samples annotated as current and reformed smokers. The status of alcohol consumption was determined using the annotations from the official PCAWG release (https://dcc.icgc.org/releases/PCAWG). The total number of clustered indels was compared in samples annotated with no alcohol consumption and those annotated as daily and weekly drinkers.

\section{Expression of driver genes}

All RNA-seq expression data were downloaded as a part of the official PCAWG release (https://dcc.icgc.org/releases/PCAWG). The relative expression data found within this release were normalized using FPKM normalization and upper quartile normalization. The relative expression of a gene was compared between those containing clustered or non-clustered events. Each distribution was then normalized to the average expression of the wild-type gene. Only genes with at least 10 total events (that is, clustered and non-clustered mutations) including at least 5 clustered events were considered for examination.

\section{SVs and clustered events}

The distance to the nearest structural variation breakpoint was calculated for each mutation in each subclass using the minimum distance to the nearest adjacent upstream or downstream breakpoint. Each distribution was modelled using a Gaussian mixture with an automatic selection criterion for the number of components ranging between one and five components using the minimum Bayesian information criteria (BIC) across all iterations. Modelling of kataegic events resulted in an optimal fit of three components, which was used to separate kataegic substitutions into SV-associated and non-SV associated mutations. DBSs and MBSs were both modelled using a single Gaussian distribution relating to non-SV associated mutations, whereas omikli and other clustered mutations were modelled using a mixture of two components, probably reflecting leakage of smaller kataegic events contributing to a weak SV-associated distribution. To account for the frequency of breakpoints across each sample, we normalized the minimum distance of each mutation to the nearest SV by calculating the expected distance between a mutation and SV for each sample using the total number of breakpoints and the overall length of a given chromosome (Extended Data Fig. 9a,b). After normalizing the kataegic events, we observed an optimal solution of two components with one SV-associated distribution (on average each mutation occurs within one-thousandth of the expected distance to nearest structural variation) and one non-SV associated distribution (on average occurring within the expected distance to the nearest structural variation). The normalized kyklonic events are consistent with the non-SV associated distribution reflecting kataegic events that occur on ecDNA typically of lengths $1-10 \mathrm{Mb}$ (ref. ${ }^{35}$ ).

\section{APOBEC $3 A$ and APOBEC $3 B$ enrichment analysis}

The enrichment score of RTCA and YTCA penta-nucleotides quantifies the frequency for which each $T p \underline{C} p A>T p \underline{K} p A$ mutation occurs at either an RTㄷA or a YTㄷA context. To account for motif availability, this score is calculated using the \pm 20 bp sequence context around each mutation and normalized by the number of cytosine bases and $\mathrm{C}>\mathrm{N}$ mutations within the set of 41-mers surrounding each mutation of interest ${ }^{7}$.

\section{APOBEC3 gene expression and kyklonas}

All RNA-seq expression data were downloaded as a part of the official PCAWG release (https://dcc.icgc.org/releases/PCAWG). The relative expression data found within this release were normalized using FPKM normalization and upper quartile normalization. The APOBEC $3 \mathrm{~A} / \mathrm{B}$ normalized expression was compared between samples containing ecDNA versus samples with no detected ecDNA and between samples with kyklonas and without kyklonas. All $P$ values were generated using a Mann-Whitney $U$-test and were corrected for multiple hypothesis testing using the Benjamini-Hochberg FDR procedure.

\section{Circular ecDNA and kataegis}

The collection of ecDNA ranges was intersected with the catalogue of clustered mutations, which was used to determine the overlapped mutational burden for each subclass of clustered event and the mutational spectra of overlapping kataegic events. Enrichments of events were calculated using statistical background models generated using SigProfilerSimulator ${ }^{51}$ that shuffled the dominant mutation in each clustered event across the genome (that is, the most frequent mutation type in a single event). The decomposed kyklonic mutational spectra were generated using the decomposition module within SigProfilerExtractor ${ }^{56}$. Only mutational signatures that increased the overall cosine similarity by at least 0.01 were used. In both the original and validation cohorts, SBS2 and SBS13 were sufficient to explain the kyklonic mutational spectra with no other known mutational signature increasing the cosine similarity by more than 0.01 . Comparisons between ecDNA with and without cancer genes were performed using the set of cancer genes from the Cancer Gene Census $(\mathrm{CGC})^{58}$. All statistical comparisons and $P$ values were calculated using a two-tailed Mann-Whitney $U$-test unless otherwise specified. For each set of tests, $P$ values were corrected for multiple hypothesis testing using the Benjamini-Hochberg FDR procedure. The predicted effect of each overlapping variant was determined using 
ENSEMBL's Variant Effect Predictor tool by reporting only the most severe consequence ${ }^{59}$.

\section{Overall survival and clustered mutations}

All survival analyses, including the generation of Kaplan-Meier curves, Cox regressions and log-rank tests, were performed using the Lifelines Python package (v.0.24.4). Across the 30 distinct whole-genome-sequenced cancer types included in the PCAWG study, only 6 cancer types contained enough samples to examine the associations between survival and overall number of clustered mutations. The sufficient sample size criteria required more than 50 samples with survival end-points with at least 30 of the samples with an observed clustered event. Each cancer type was analysed separately by comparing the survival of samples with a high clustered mutational burden (top 80th percentile across a given cancer type) to the survival of samples with a low clustered mutational burden (bottom 20th percentile across a given cancer type).

Analysis of whole-exome-sequenced samples from TCGA was altered to reflect the limited resolution for identifying clustered mutations within the exome. Specifically, SigProfilerSimulator (v.1.0.2 $)^{51}$ was used to derive an IMD cut-off for each sample based on the TMB within the exome and the mutational patterns for a given sample. Mutations were randomly shuffled while maintaining the mutational burden within the exome of each chromosome, the \pm 2 bp sequence context for each mutation and the transcriptional strand bias ratios across all mutations. Each sample was simulated 100 times and an IMD cut-off was calculated using the same methods as outlined for the detection of clustered events within PCAWG. Owing to the limited number of detected events, 22 cancer types had sufficient data to perform survival analysis. Each cancer type was analysed separately by comparing samples with at least a single clustered event to samples with no detected clustered events within the exome.

For both PCAWG and TCGA analyses, survival distributions within a given cancer type were compared using a log-rank test. Cox regressions were performed to determine hazards ratios and to correct for age and total mutational burden. All $P$ values were also corrected for multiple hypothesis testing using the Benjamini-Hochberg FDR procedure.

To investigate differential survival associated with the detection of clustered events within cancer driver genes, Kaplan-Meier survival curves were compared between individuals with clustered versus non-clustered mutations within a given cancer driver gene. The distributions were compared using a log-rank test. Cox regressions were performed to determine the hazards ratios and to correct for age, total mutational burden and cancer type across TCGA. Cox regressions performed for the MSK-IMPACT cohort were corrected for total mutational burden and cancer type. No corrections were performed for age as these metadata were not available for the MSK-IMPACT cohort. All $P$ values were also corrected for multiple hypothesis testing using the Benjamini-Hochberg FDR procedure.

\section{Validation of kyklonas in three cohorts}

All three validation cohorts were analysed analogous to the PCAWG cohorts. Specifically, clustered mutations were classified by calculating a sample-dependent IMD threshold for clustered versus non-clustered mutations using a background model generated by SigProfilerSimulator $^{51}$. All clustered mutations were subclassified into DBS, MBS, omikli, kataegis or other mutations. AmpliconArchitect (v.1.2) was used to determine regions of focal amplifications ${ }^{60}$, which were used for subsequent validation of kyklonic events by overlapping kataegic events with all detected focal amplifications. The decomposed kyklonic mutational spectra were generated using the decomposition module within SigProfilerExtractor ${ }^{56}$. Only mutational signatures that increased the overall cosine similarity by at least 0.01 were used. In both the original and validation cohorts, SBS2 and SBS13 were sufficient to explain the kyklonic mutational spectra with no other known mutational signature increasing the cosine similarity by more than 0.01 .

\section{Cancer-type abbreviations}

Biliary-AdenoCA, biliary adenocarcinoma; Bladder-TCC, bladder transitional cell carcinoma; Bone-Epith, bone epithelioid; Bone-Leiomyo, bone leiomyosarcoma; Bone-Osteosarc, bone osteosarcoma; Breast-AdenoCA, breast adenocarcinoma; Breast-LobularCA, breast lobular carcinoma; CNS-GBM, glioblastoma (central nervous system); CNS-Medullo, medulloblastoma (central nervous system); CNS-Oligo, oligodendroglioma (central nervous system); CNS-PiloAstro, pilocytic astrocytoma (central nervous system); Cervix-AdenoCA, cervix adenocarcinoma; Cervix-SCC, cervix squamous cell carcinoma; ColoRect-AdenoCA, colorectal adenocarcinoma; Head-SCC, head and neck squamous cell carcinoma; Kidney-ChRCC, chromophobe renal cell carcinoma; Kidney-RCC, renal cell carcinoma; Liver-HCC, hepatocellular carcinoma; Lung-AdenoCA, lung adenocarcinoma; Lung-SCC, lung squamous cell carcinoma; Lymph-BNHL, B-cell nonHodgkin lymphoma; Lymph-CLL, chronic lymphocytic leukaemia; Lymph-NOS, metastatic lymphoma; Myeloid-AML, acute myeloid leukaemia; Myeloid-MPN, myeloproliferative neoplasm; Oeso-AdenoCA, oesophageal adenocarcinoma; Ovary-AdenoCA, ovary adenocarcinoma; Panc-AdenoCA, pancreatic adenocarcinoma; Panc-Endocrine, pancreatic neuroendocrine carcinoma; Prost-AdenoCA, prostate adenocarcinoma; Skin-Melanoma, malignant melanoma; StomachAdenoCA, stomach adenocarcinoma; Thy-AdenoCA, thyroid adenocarcinoma; Uterus-AdenoCA, uterine adenocarcinoma.

\section{Reporting summary}

Further information on research design is available in the Nature Research Reporting Summary linked to this paper.

\section{Data availability}

No data were generated specifically for this study. All data were and can be downloaded from the appropriate links, repositories and references. Specifically, for the discovery cohort, all data and metadata were obtained from the official PCAWG release (https:// dcc.icgc.org/releases/PCAWG). All data and metadata for TCGA samples were obtained from the GDC (https://gdc.cancer.gov/). Genomics data for clonally expanded cell lines were downloaded from the European Genome-phenome Archive (EGAD00001004201, EGAD00001004203 and EGAD00001004583). For the three validation cohorts, datasets were downloaded as submitted by the original publications and genomics data were downloaded from their respective repositories: EGAD00001004162 for 61 undifferentiated sarcomas ${ }^{44}$ (European Genome-phenome Archive); EGAD00001006868 for 187 high-confidence oesophageal squamous cell carcinomas ${ }^{46}$ (European Genome-phenome Archive); and phs001697.v1.p1 for 280 lung adenocarcinomas ${ }^{45}(\mathrm{dbGaP})$. Somatic mutations and metadata for the MSK-IMPACT Clinical Sequencing Cohort composed of 10,000 clinical cases $^{42}$ were downloaded from cBioPortal (https://www.cbioportal. org/study/summary?id=msk_impact_2017).

\section{Code availability}

The SigProfiler compendium of tools are developed as Python packages and are freely available for installation through PyPI or directly through GitHub (https://github.com/AlexandrovLab/). For all tools, each package is fully functional, free and open sourced distributed under the permissive 2-Clause BSD License and is accompanied by extensive documentation: (1) SigProfilerMatrixGenerator ${ }^{55}$ (v.1.2.0; https://github.com/AlexandrovLab/SigProfilerMatrixGenerator);(2) SigProfilerSimulator ${ }^{51}$ (v.1.0.2; https://github.com/AlexandrovLab/ 
SigProfilerSimulator); and (3) SigProfilerExtractor ${ }^{56}$ (v.1.1.0; https:// github.com/AlexandrovLab/SigProfilerExtractor). Each SigProfiler tool also has an R wrapper available for installation through the GitHub repositories. AmpliconArchitect ${ }^{34}$ (v.1.2) is also freely available and can downloaded from https://github.com/virajbdeshpande/AmpliconArchitect. The core computational pipelines used by the PCAWG Consortium for alignment, quality control and variant calling are available to the public at https://dockstore.org/search?search=pcawg under the GNU General Public License v.3.0, which allows for reuse and distribution.

51. Bergstrom, E. N., Barnes, M., Martincorena, I. \& Alexandrov, L. B. Generating realistic null hypothesis of cancer mutational landscapes using SigProfilerSimulator. BMC Bioinf. 21, 438 (2020)

52. Hess, J. M. et al. Passenger hotspot mutations in cancer. Cancer Cell 36, 288-301 (2019)

53. Polak, P. et al. Cell-of-origin chromatin organization shapes the mutational landscape of cancer. Nature 518, 360-364 (2015).

54. Amemiya, H. M., Kundaje, A. \& Boyle, A. P. The ENCODE Blacklist: identification of problematic regions of the genome. Sci. Rep. 9, 9354 (2019).

55. Bergstrom, E. N. et al. SigProfilerMatrixGenerator: a tool for visualizing and exploring patterns of small mutational events. BMC Genomics 20, 685 (2019).

56. Islam, S. M. A. et al. Uncovering novel mutational signatures by de novo extraction with SigProfilerExtractor. Preprint at https://doi.org/10.1101/2020.12.13.422570 (2020).

57. Polak, P. et al. A mutational signature reveals alterations underlying deficient homologous recombination repair in breast cancer. Nat. Genet. 49, 1476-1486 (2017).

58. Sondka, Z. et al. The COSMIC Cancer Gene Census: describing genetic dysfunction across all human cancers. Nat. Rev. Cancer 18, 696-705 (2018).

59. McLaren, W. et al. The Ensembl Variant Effect Predictor. Genome Biol. 17, 122 (2016).

60. Deshpande, V. et al. Exploring the landscape of focal amplifications in cancer using AmpliconArchitect. Nat. Commun. 10, 392 (2019).

Acknowledgements E.N.B. and L.B.A. were supported by the Cancer Research UK Grand Challenge Award C98/A24032 as well as US National Institute of Health (NIH) grants R01ES030993-01A1 and R01ES032547. L.B.A. is an Abeloff V Scholar and he was also supported by an Alfred P. Sloan Research Fellowship. Research at the University of California
San Diego was also supported by a Packard Fellowship for Science and Engineering to L.B.A. N.P. is funded through a Cancer Research UK grant (grant no. 18387) and is supported by the UCLH Biomedical Research Centre and the Cancer Research UK Experimental Cancer Centre. C.D.S. is funded through Cancer Research UK and the Neurofibromatosis Research Initiative (NFRI) at Boston Children's Hospital-GeM consortium. M.P. is supported by a European Molecular Biology Organization (EMBO) Long-Term Fellowship (ALTF 760-2019). V.B. and J.L. were supported in part by grants U24CA264379 and R01GM114362 from the NIH. P.S.M. is supported in part by grants U24CA264379 and RO1CA238249 from the NIH. Cancer research in the R.S.H. laboratory is supported by NCl grant P01CA234228. R.S.H. is the Margaret Harvey Schering Land Grant Chair for Cancer Research, a Distinguished University McKnight Professor and an Investigator of the Howard Hughes Medical Institute. The funders had no roles in study design, data collection and analysis, decision to publish or preparation of the manuscript.

Author contributions E.N.B. and L.B.A. designed the overall study. E.N.B. performed all genomics analyses with help from J.L., M.P., A.K., M.B., T.Z., C.D.S., N.P., M.T.L., V.B., P.S.M. R.S.H. and L.B.A. Specifically, J.L., V.B., A.K. and P.S.M. assisted in analysis and discussion of ecDNA. M.P. and R.S.H. aided in the analysis and interpretation of APOBEC3 mutational signatures. A.K., M.B., T.Z., C.D.S., N.P. and M.T.L. gathered the validation cohorts and helped with the subsequent computational validation analyses. E.N.B. performed all clinical association analysis and all analysis of gene expression. E.N.B. and L.B.A. wrote the manuscript with help and input from all other authors. All authors read and approved the final manuscript.

Competing interests M.P. is a shareholder in Vertex Pharmaceuticals. V.B. is a co-founder, consultant and Scientific Advisory Board member of, and has equity interest in, Boundless Bio, and Abterra. The terms of this arrangement have been reviewed and approved by the University of California San Diego in accordance with its conflict-of-interest policies. E.N.B. and L.B.A. declare filing a provisional patent application for using clustered mutations as clinical prognostic biomarkers in cancer. P.S.M. is a co-founder of Boundless Bio. He has equity in the company and he chairs the Scientific Advisory Board, for which he is compensated. L.B.A. is an inventor on US patent no. $10,776,718$ for source identification by non-negative matrix factorization. All other authors declare no competing interests.

Additional information

Supplementary information The online version contains supplementary material available at https://doi.org/10.1038/s41586-022-04398-6.

Correspondence and requests for materials should be addressed to Ludmil B. Alexandrov. Peer review information Nature thanks the anonymous reviewers for their contribution to the peer review of this work.

Reprints and permissions information is available at http://www.nature.com/reprints. 
a

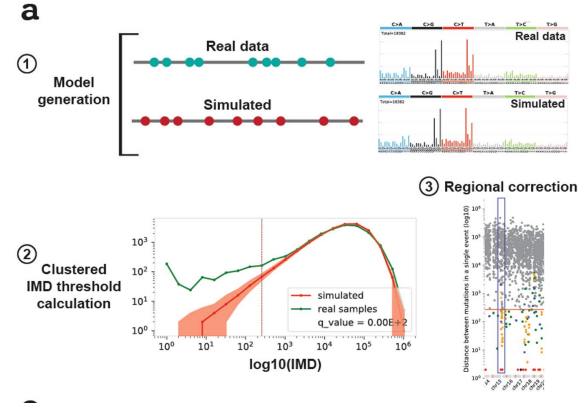

e

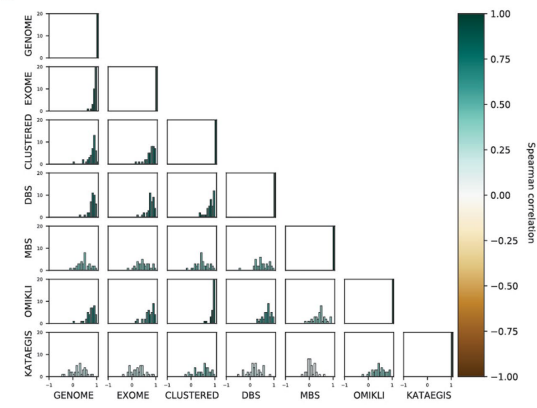

g

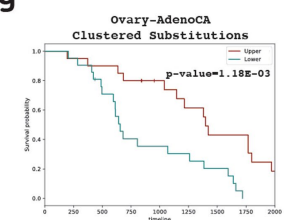

i
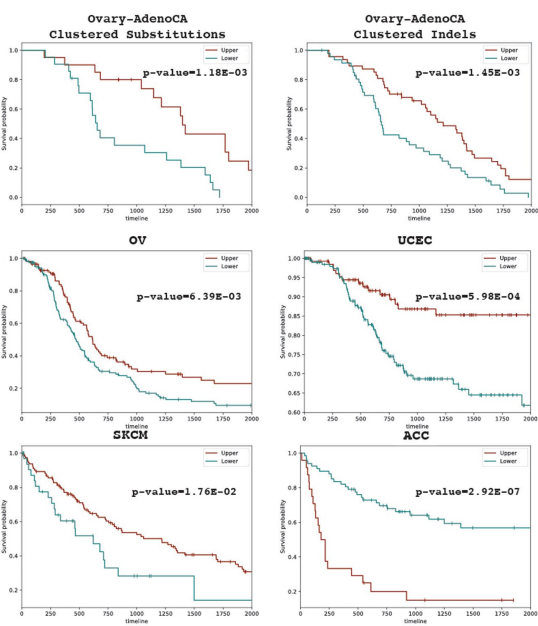

UCEC
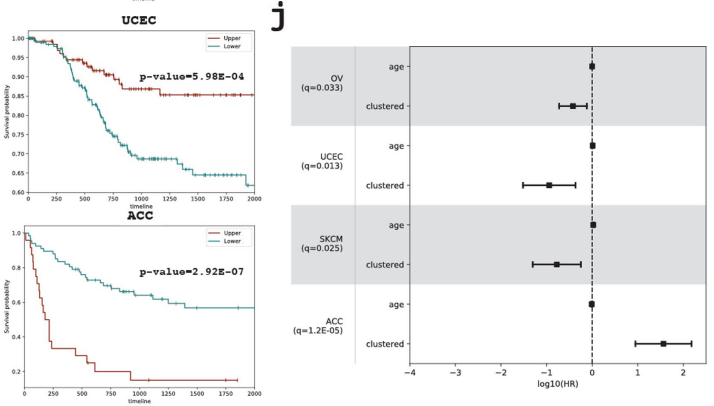

d
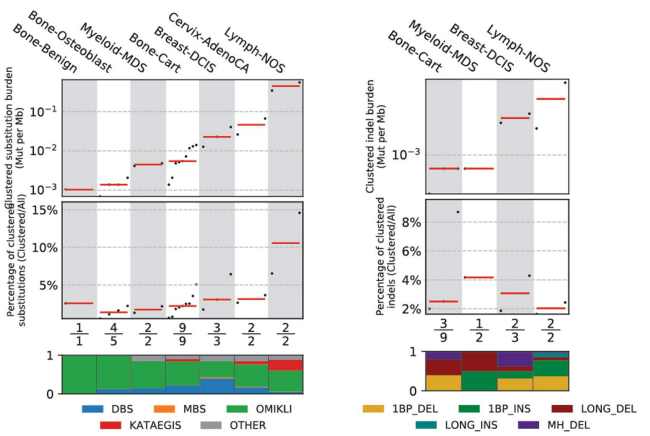

$\mathbf{f}$

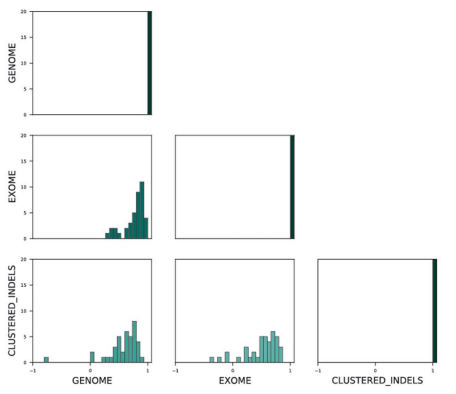

h
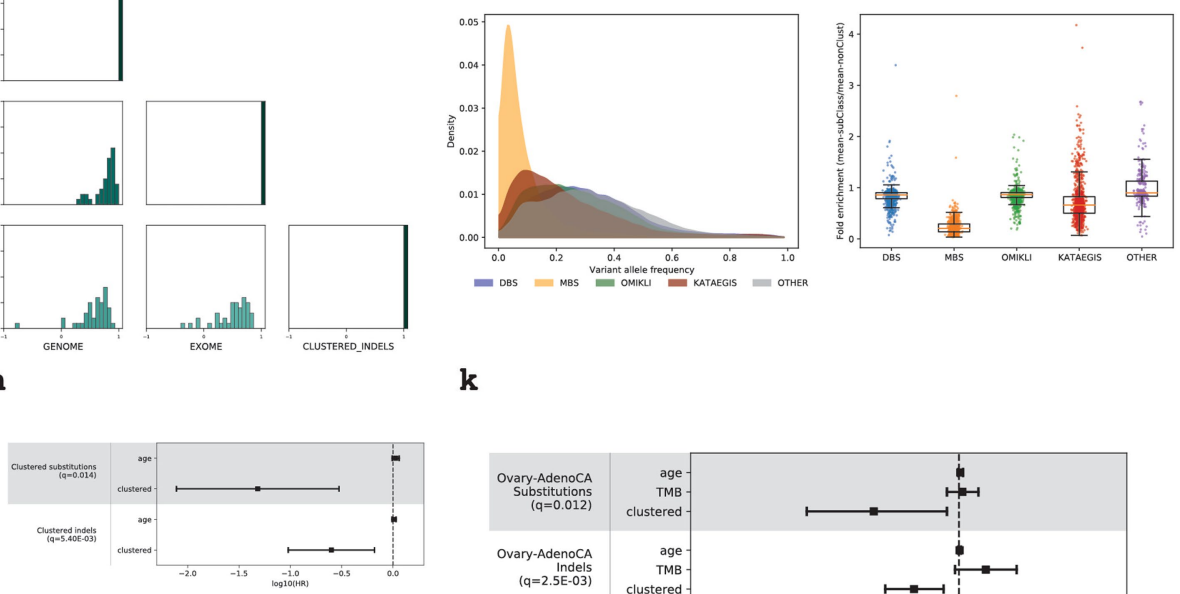

$\mathbf{k}$
Extended Data Fig. 1 | Identification and clinical associations of clustered events. a, Schematic depiction for separating clustered mutations for a sample. b, Subclassification of clustered substitutions and indels. Expected IMD derived using steps 2 and 3 (a).c, Distribution of indels present in a single clustered event. d, Distribution of clustered substitutions (left) and indels (right) across cancers with less than 10 samples subclassified into different categories. e, Correlations between TMB of each sample, the TMB within the exome, or the TMB for each class of clustered substitutions (left) and indels (right).f, Distribution of VAFs for all clustered substitution classes (left; DBS: 1,215 samples; MBS: 851; omikli:1,466; kataegis:1,108; other:335) with the average fold enrichment compared against non-clustered mutations (right). For each boxplot, the middle line reflects the median, the lower and upper bounds correspond to the first and third quartiles, and the lower and upper whiskers extend from the box by $1.5 x$ the inter-quartile range (IQR). g, KaplanMeier curves between samples with high (top $80^{\text {th }}$ percentile) and low (bottom $20^{\text {th }}$ percentile) clustered substitution (left) or indel (right) burdens in PCAWG ovarian cancer. $\mathbf{h}$, Cox regressions performed for PCAWG cancer types while correcting for age ( $n=20$ upper and $n=21$ lower clustered substitutions; $n=49$ upper and $n=49$ lower clustered indels). $\mathbf{i}$, Kaplan-Meier survival curves for TCGA cancer types with a differential patient outcome associated with the detection of any clustered mutations. $\mathbf{j}, \mathbf{k}$, Cox regressions performed for TCGA samples while correcting for age $(\mathbf{j})$ and total mutational burden $(\mathbf{k})(\mathrm{OV}$ : $\mathrm{n}=111$ upper, $\mathrm{n}=159$ lower clustered substitutions; UCEC: $\mathrm{n}=322$ upper, $\mathrm{n}=64$ lower; ACC: $n=24$ upper, $n=67$ lower). PCAWG ovarian cancers were included in $\mathbf{k}$. Centre of measure for each Cox regression reflects the $\log _{10}$ (Hazards ratios) with the $95 \%$ confidence intervals in $\mathbf{h}-\mathbf{k}$ ). 
a
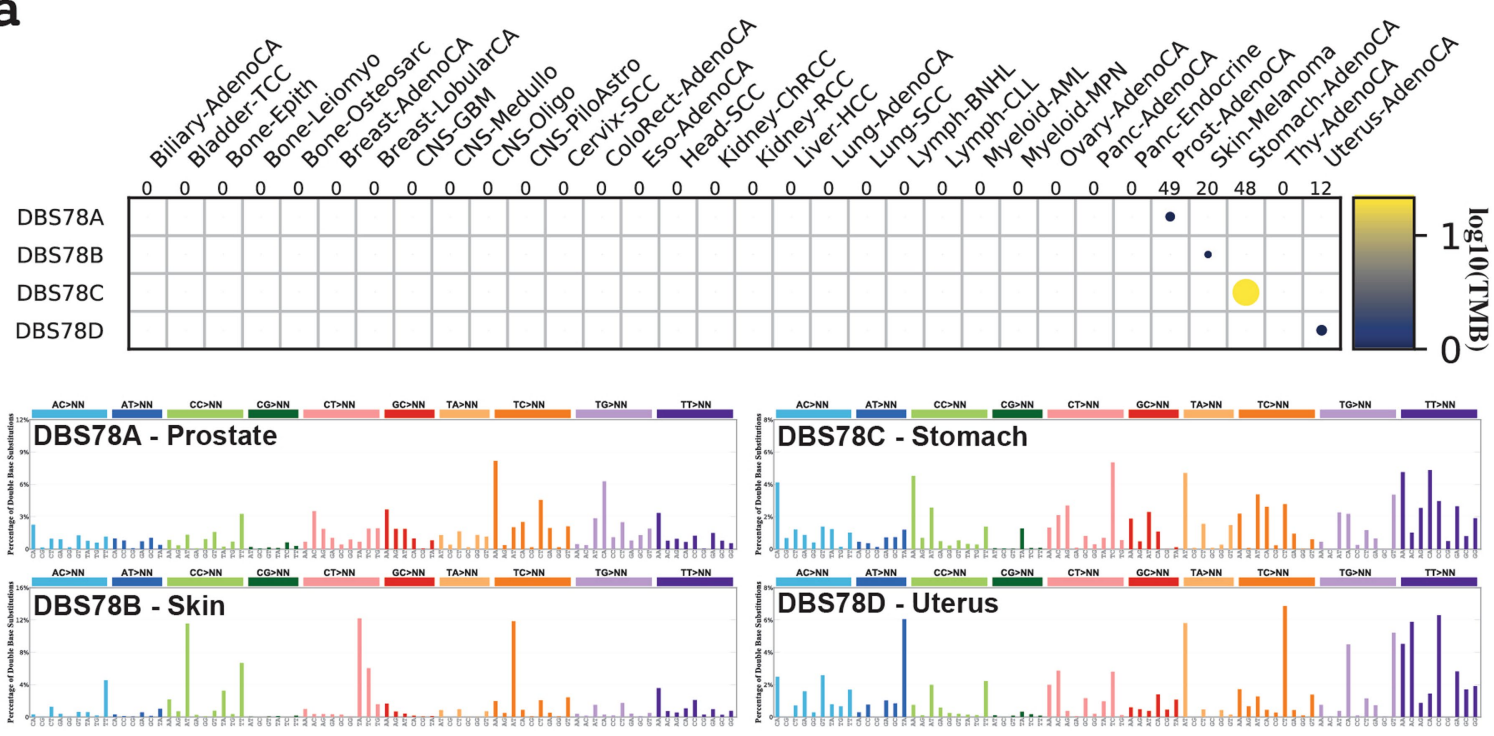

b

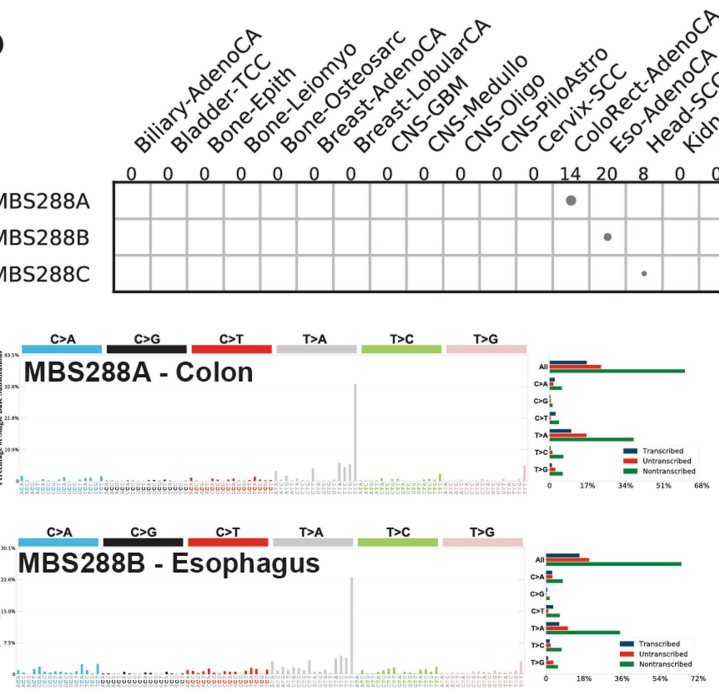

Extended Data Fig. 2 | De novo signatures of DBS and MBS signatures. a, The activity of DBS de novo signatures (top) and the corresponding signatures extracted from prostate, skin, stomach, and uterine cancers that could not be accurately reconstructed using known COSMIC mutational signatures

(bottom; Methods).b, The activity of MBS de novo signatures (top) and the corresponding signatures extracted from colon, oesophagus, and head and neck cancers that could not be accurately reconstructed using known COSMIC mutational signatures (bottom; Methods). 
a
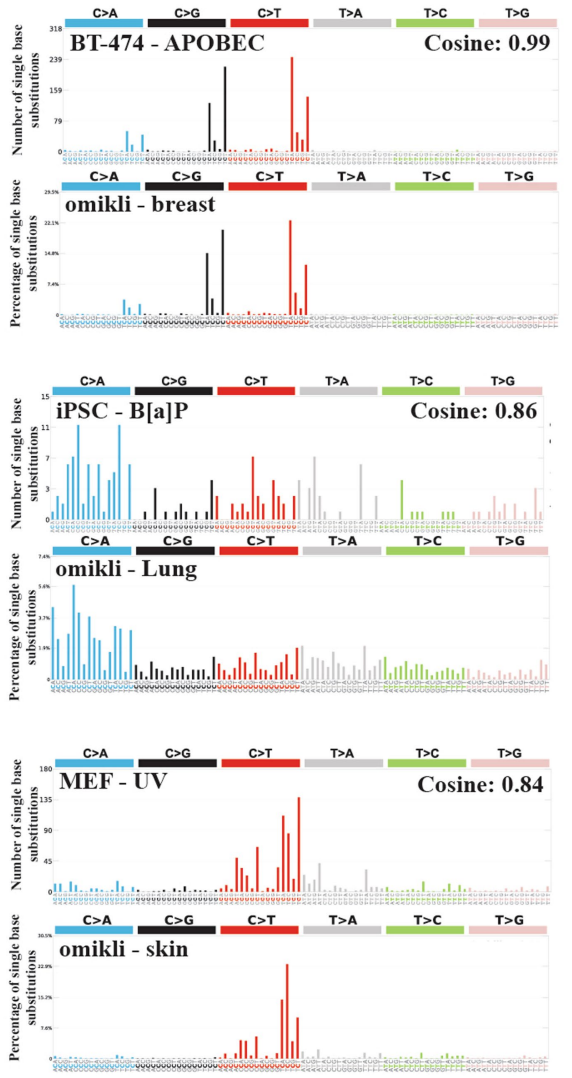

b

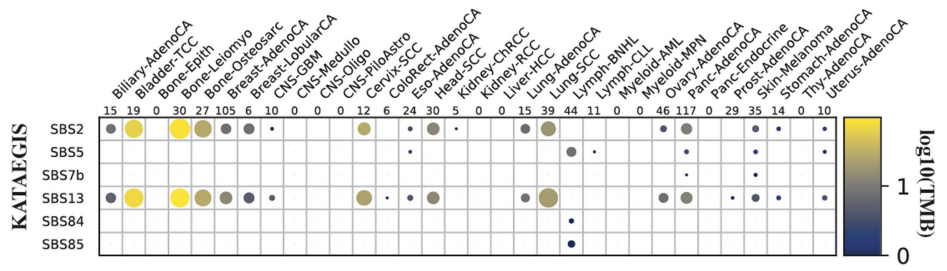

C
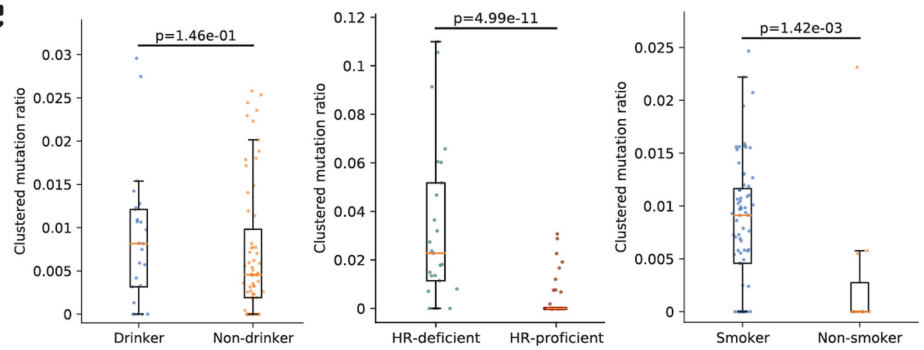

d

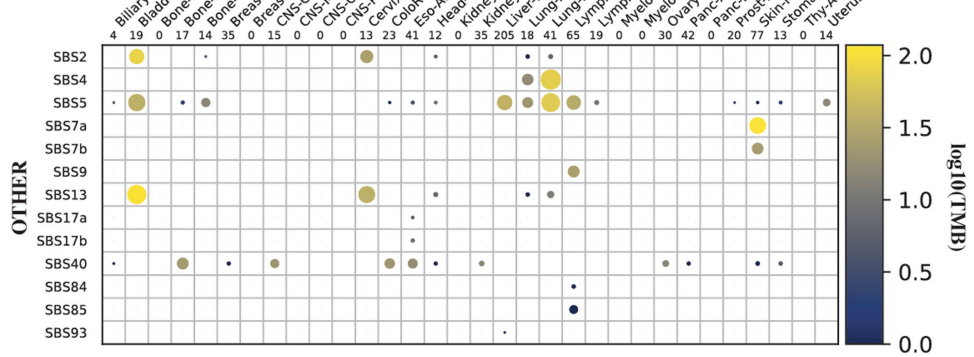

Extended Data Fig. 3 | Experimental validation and epidemiological associations of clustered mutational processes. a, Experimental validation of three omikli processes. Specifically, APOBEC3-associated omikli were validated using a clonally expanded BT- 474 breast cancer cell line (top), omikli events resulting from exposure to benzo[ $a]$ pyrene were validated using iPS cells (middle), and omikli events resulting from exposure to ultraviolet light were validated using iPS cells (bottom). b, Mutational processes of strand-coordinated kataegic events. c, Epidemiological associations comparing the ratio of clustered TMB to the total TMB for a given sample between: drinkers $(n=25)$ and non-drinkers $(n=61)$; smokers $(n=68)$ and non-smokers ( $\mathrm{n}=11)$; homologous-recombination deficient (HR-deficient; $\mathrm{n}=25$ ) and homologous-recombination proficient samples (HR-proficient; $n=64$ ). For each boxplot, the middle line reflects the median, the lower and upper bounds of the box correspond to the first and third quartiles, and the lower and upper whiskers extend from the box by $1.5 x$ the inter-quartile range (IQR). P-values were calculated using a two-tailed Mann-Whitney $U$-test.

d, Mutational processes of clustered events with inconsistent VAFs classified as other clustered substitutions. A minimum of two samples are required per cancer type for visualization (Methods). 


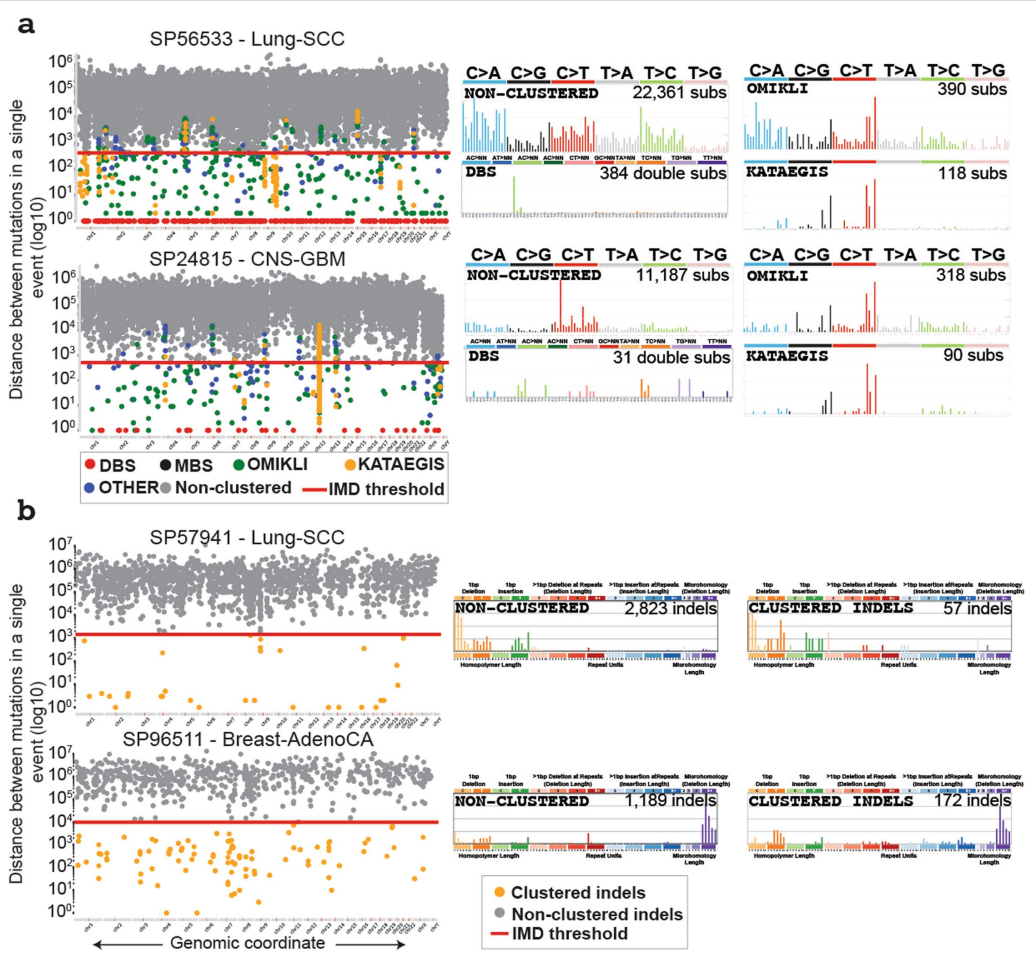

Extended Data Fig. 4 | Examples of clustered mutational signatures. a, Two samples depicting the intra-mutational distance (IMD) distributions of substitutions across genomic coordinates, where each dot represents the minimum distance to adjacent mutations for a selected mutation coloured based on the corresponding subclassification of event (rainfall plot; left). The red lines depict the sample-dependent IMD threshold for each sample. Specific clustered mutations may be above this threshold based on corrections for regional mutation density. The mutational spectra for the different catalogues of clustered and non-clustered substitutions for each sample (right; MBS are not shown). $\mathbf{b}$, Two samples illustrating the IMD distributions of indels across the given genomes, with the IMD indel thresholds shown in red (left). The non-clustered and clustered indel catalogues for each sample (right). 


\section{Article}

a
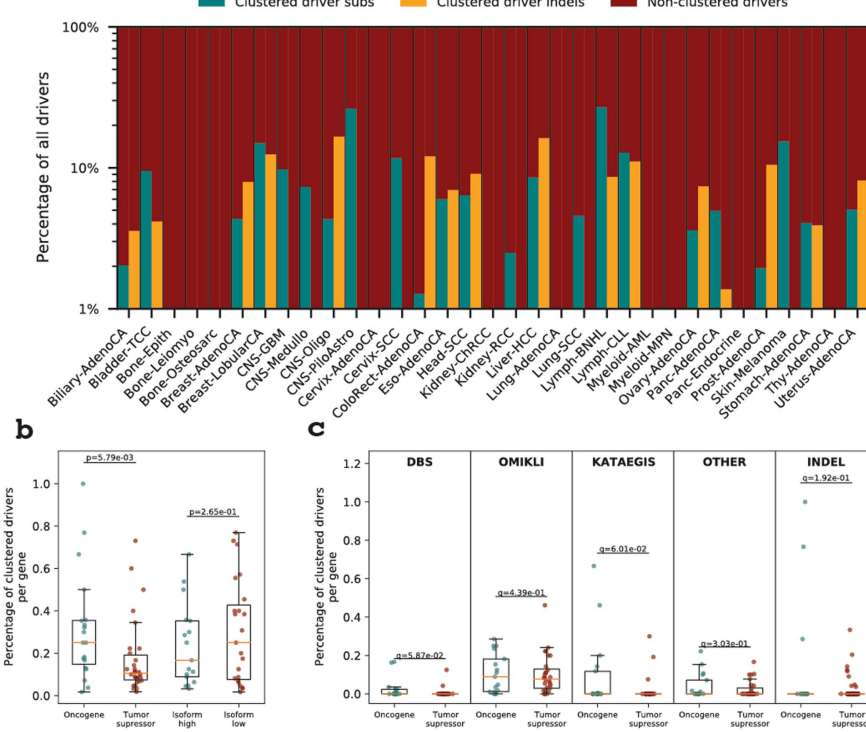

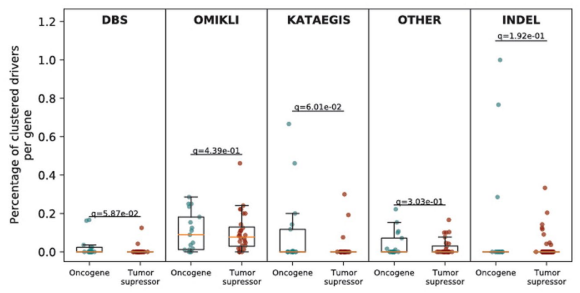

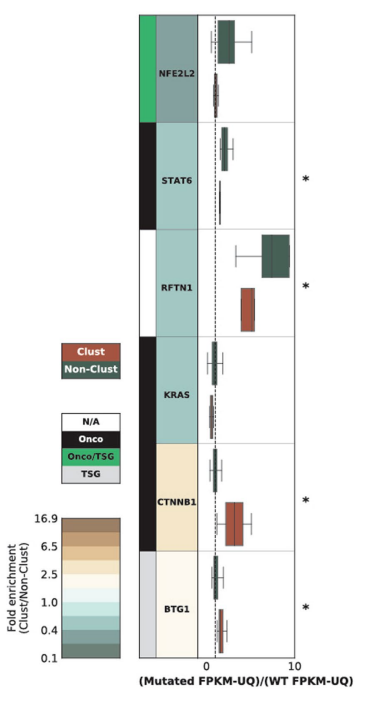
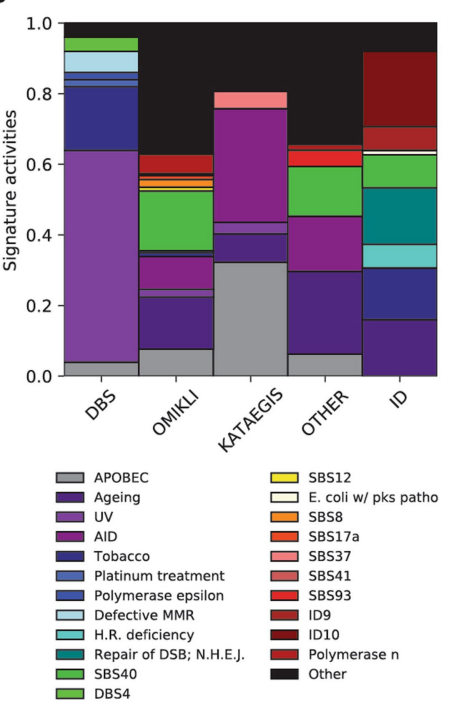

Extended Data Fig. 5 | Mutational processes of clustered driver events. a, The percentage of clustered driver substitutions and indels within each cancer type. All samples 2,583 whole-genome sequenced samples from PCAWG with a detected driver event are included; however, cancer types with fewer than 10 samples are not presented. $\mathbf{b}$, The proportion of clustered driver mutations per cancer gene compared between oncogenes ( $n=19$ genes) versus tumour suppressor genes ( $\mathrm{n}=30$ genes) and genes with high numbers of isoforms $(n=17)$ versus genes with low numbers of isoforms $(n=23$; upper and lower quartiles of isoforms across all cancer drivers). c, The proportion of clustered driver mutations for a given subclass per cancer gene compared between oncogenes $(n=17$ genes with clustered substitutions and $n=13$ with for clustered indels) versus tumour suppressor genes $(n=28$ genes with clustered substitutions and $n=70$ genes with clustered indels). $\mathbf{d}$, The relative

expression of driver genes containing clustered (copper) versus non-clustered events (green). All expression values were normalized using FPKM normalization and upper quartile normalization obtained from the official PCAWG release and were subsequently normalized using the average expression of the wild-type gene. A value of 1 (dashed lined) reflects no difference in expression compared to the wild-type gene.e, The proportional activity of mutational signatures contributing to clustered driver events within each subclass. MBSs did not contribute to any reported driver events. For analyses in $\mathbf{b}-\mathbf{d}$, $\mathrm{p}$-values were generated using a two-tailed Mann-Whitney $U$-test $\left({ }^{*} \mathrm{P}<0.05 ; \mathrm{p}=0.03\right.$ for STAT6; $\mathrm{p}=0.04$ for CTNNB1; $\mathrm{p}=0.02$ for BTG1). For each boxplot, the middle line reflects the median, the lower and upper bounds of the box correspond to the first and third quartiles, and the lower and upper whiskers extend from the box by $1.5 x$ the inter-quartile range (IQR). 


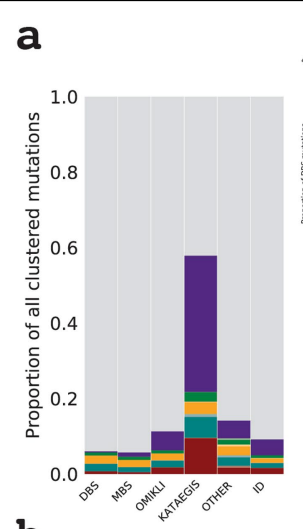

b
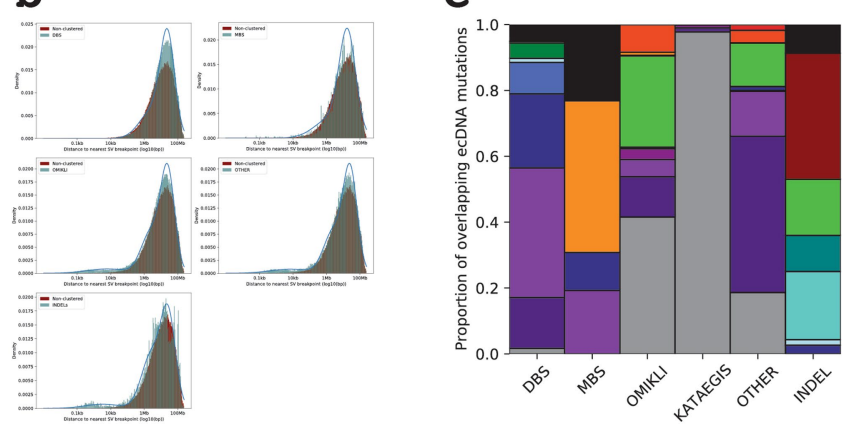

Extended Data Fig. 6 | Clustered events and structural variations. a, The proportion of all clustered events co-locating with structural variations across all cancer types (left) and across each cancer type (right). b. The distance to the nearest structural variation for each class of clustered mutations (teal), and non-clustered mutations (red). The distribution for each class of clustered events were modelled using a Gaussian mixture (blue line). DBSs and MBSs were modelled using a single distribution, whereas omikli, other, and indels were modelled using two components reflecting the minimal distribution of overlap with structural variations. c, The mutational signatures active in
MBS

OMIKLI

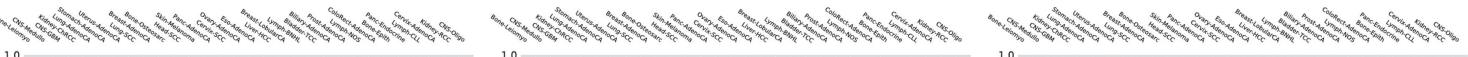

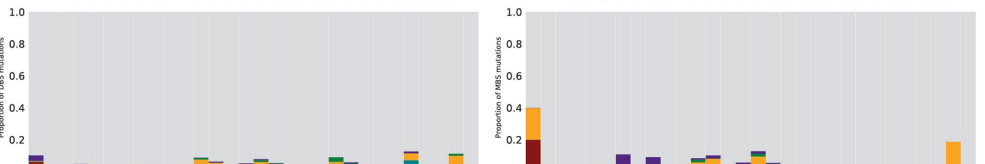

OTHER

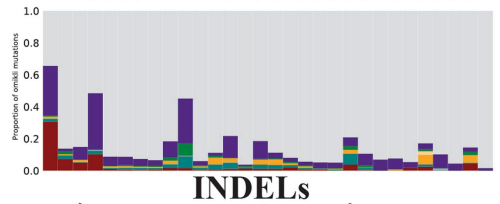

Ton
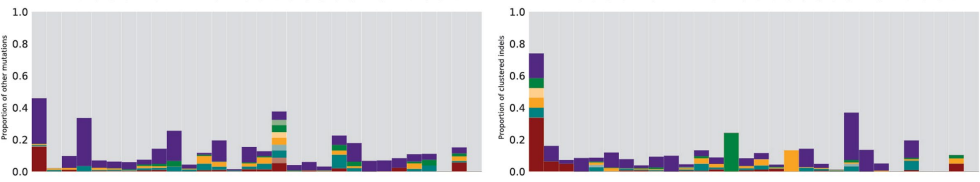

d

non-ecDNA kataegis (Pan-cancer)
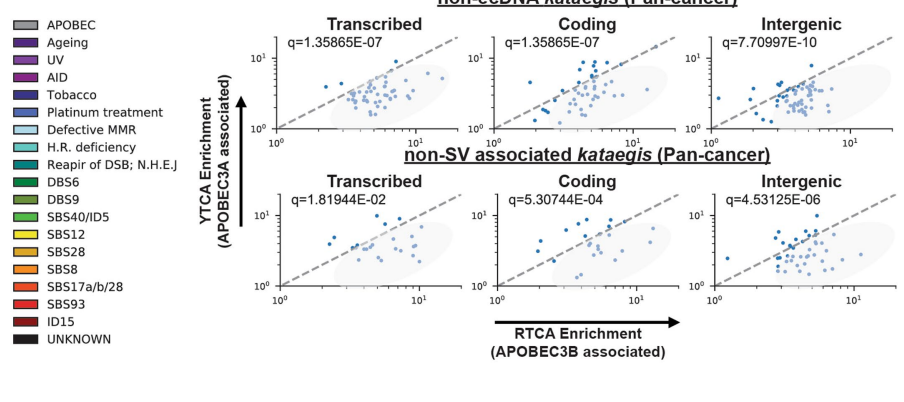

ecDNA clustered events. d, YTㅁA versus RTㅁA enrichments per sample within non-ecDNA kataegis (top) and non-SV associated kataegis (bottom), where YTㅡA and RT $\underline{C} A$ enrichment is suggestive of APOBEC 3 A or APOBEC $3 B$ activity, respectively. Genic mutations were divided into transcribed (template strand) and coding mutations. The RTㅡA/YTㅁA fold enrichments were compared to the fold enrichments of non-clustered mutations ( $p$-values calculated using two-tailed Mann-Whitney $U$-tests and corrected for multiple hypothesis testing using the Benjamini-Hochberg FDR procedure). 
a

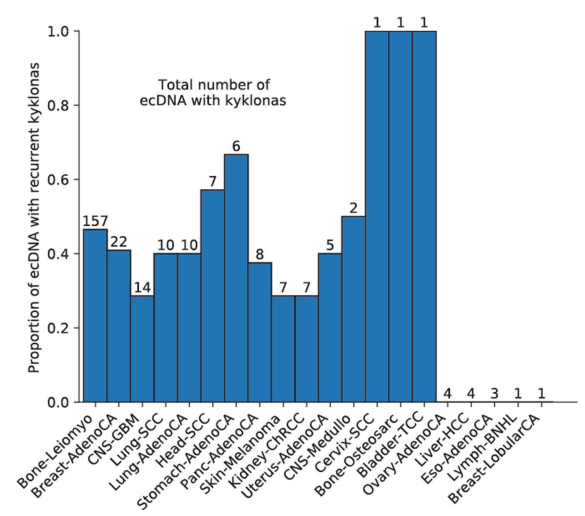

C
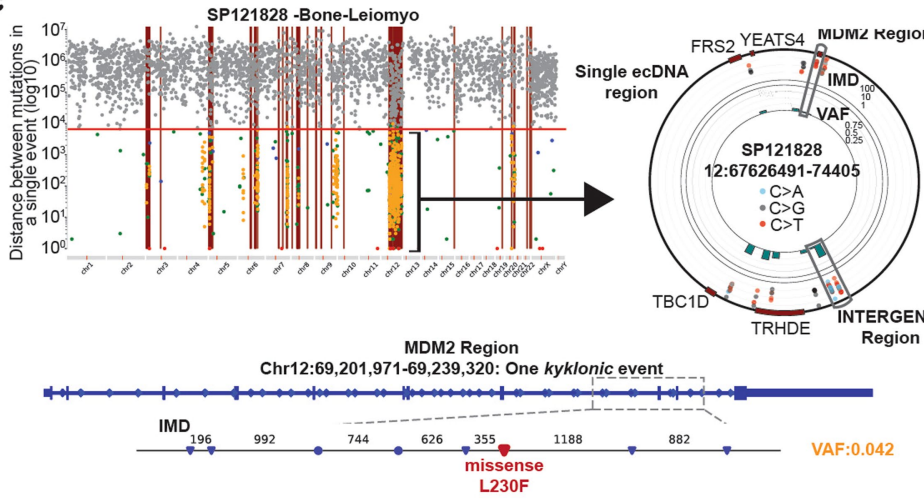

INTERGENIC Region

Chr12:73,331,203-73,404,813: Two kyklonic events

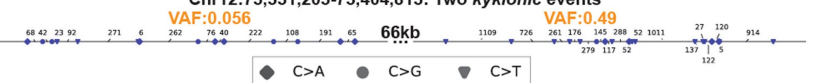

d

$$
\rightarrow C>A
$$

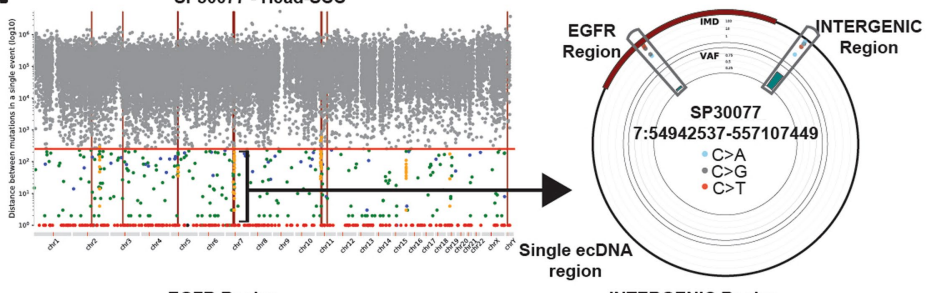

EGFR Region

Chr7:55,086,971-55,273,310: One kataegic event

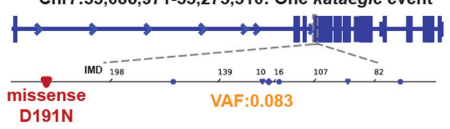

Extended Data Fig. $7 \mid$ Recurrent mutagenesis and functional effects of

kyklonas. a, The total number of recurrently mutated ecDNA displayed as a proportion of the total number of ecDNA with kyklonas for a given cancer type. The total number of ecDNA with kyklonas are displayed above each bar plot for each cancer type. All ecDNA with recurrent hypermutation were considered enriched for kyklonic events after correcting for multiple hypothesis testing (Z-score test; q-values $<0.05$ ). b, Proportion of samples containing ecDNA divided exclusively into those with co-occurring kataegis, no kataegis overlap, and no detected kataegis across the entire genome. The number of samples included in each cancer type are listed. For certain cancer types, as few as a single sample may represent the entire proportional breakdown (for example, Bone-Osteosarc or Bone-Epith).c, A single sarcoma genome and d, a single head squamous cell carcinoma genome depicting the overlap of kataegis with ecDNA regions displayed as a rainfall (top left) with a single zoomed in ecDNA represented using a circos plot (top right). Bottom: Two regions of the ecDNA with overlapping kyklonic events. VAFs are shown per event (orange). e, Kyklonic substitutions resulting in recurrent coding mutations within known cancergenes. 
a
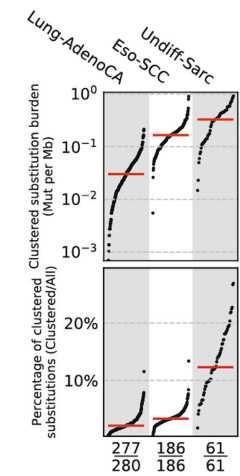

Dי

DBS
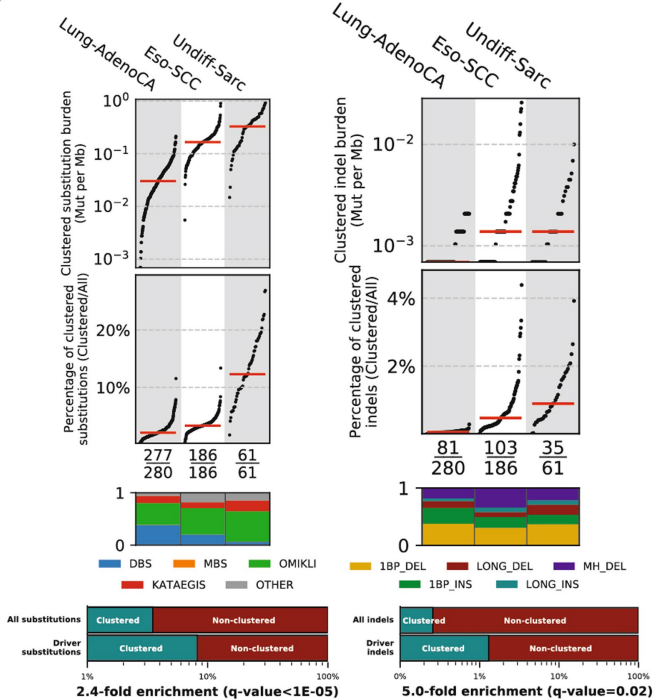

b
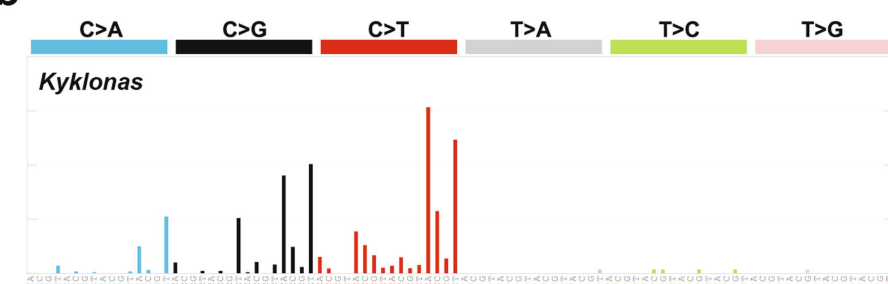

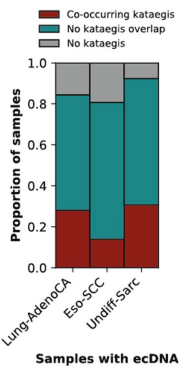

d

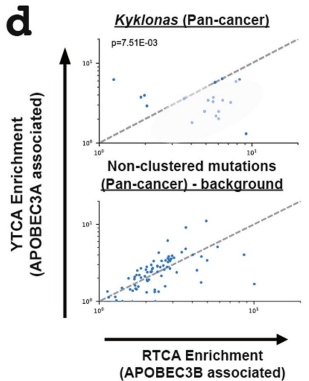

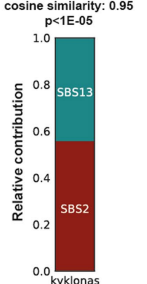

e

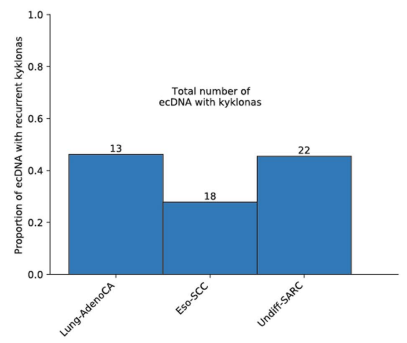

Extended Data Fig. 8 | Validation of APOBEC3 hypermutation of ecDNA in three independent cohorts. a, Distribution of clustered substitutions (left) and clustered indels (right) across three validation cohorts. Clustered substitutions were subclassified into DBSs, MBSs, omikli, kataegis, and other clustered mutations. Top: Each black dot represents a single cancer genome. Red bars reflect the median clustered TMB and the percentage of clustered mutations contributing to the overall TMB of a given sample for each cancer type. Middle: The proportion of each subclass of clustered events for a given cancer type with the total number of samples having at least a single clustered event over the total number of samples within a given cancer cohort. Bottom: Percentage of clustered mutations compared to the percentage of clustered driver events for substitutions (left) and indels (right). P-values were calculated using a Fisher's exact test and corrected for multiple hypothesis testing using
Benjamini-Hochberg FDR procedure. b, Left: The mutational spectrum of all kyklonas across the validation cohorts. Right: The proportion of kyklonic events attributed to SBS2 and SBS13 (p-value determined using a Z-score test; Methods).c, The proportion of samples with ecDNA that co-occur with kataegis, do not co-occur with kataegis, or do not have any detected kataegic activity across each cohort. d, YTC $\underline{A}$ versus RTCA enrichments per sample with kyklonas, where YTC $\underline{A}$ and RT $\underline{A} A$ enrichment is suggestive of higher APOBEC $3 \mathrm{~A}$ or APOBEC3B activity, respectively. The RTㅡA/YTㅁA fold enrichments were compared to the fold enrichments of non-clustered mutations ( $p$-values calculated using a two-tailed Mann-Whitney $U$-test).e, The proportion of ecDNA with kyklonas that contain multiple kyklonic events. The total number of ecDNA with kyklonas are displayed above each bar plot for each cancer type. 


\section{Article}

a

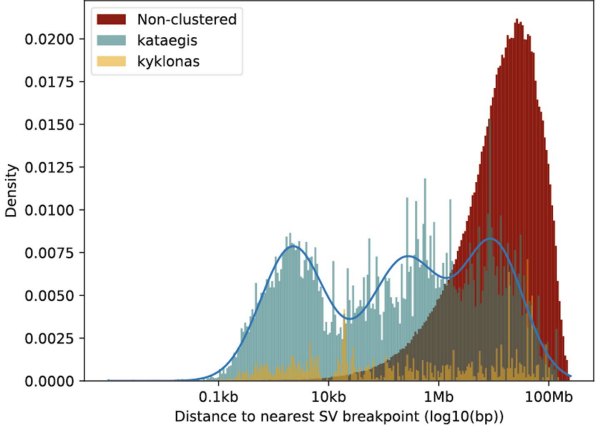

\section{b}

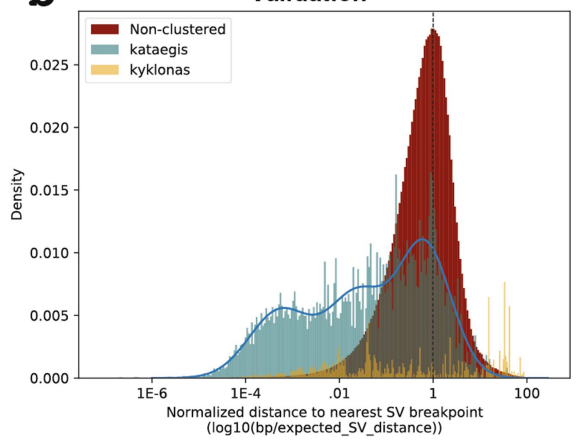

PCAWG

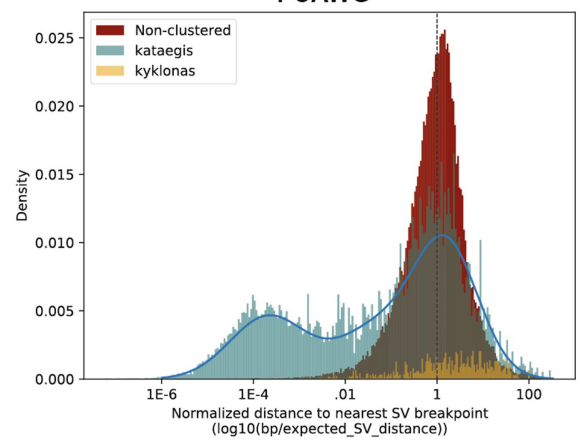

Extended Data Fig. 9|Kyklonas occur distally from structural breakpoints across three independent cohorts. a, The distance to the nearest breakpoint for all kataegic mutations (teal), kyklonas (gold), and non-clustered mutations (red) across the three validation cohorts. b, Distances to the nearest SV breakpoints were normalized by calculating the expected distance a mutation would fall from a breakpoint given the number of breakpoints detected per chromosome and the overall length of the chromosome across the validation cohorts (left) and PCAWG (right). A value of 1 (dashed line) reflects a distance that one would expect based on the random placement of a mutation across the chromosome, whereas a value less than 1 reflects a mutation occurring closer than what is expected by random chance. The distributions of kataegic mutations were modelled using Gaussian mixture models (blue lines) with an automatic selection criterion for the number of components using the minimum Bayesian information criteria (BIC). 
a

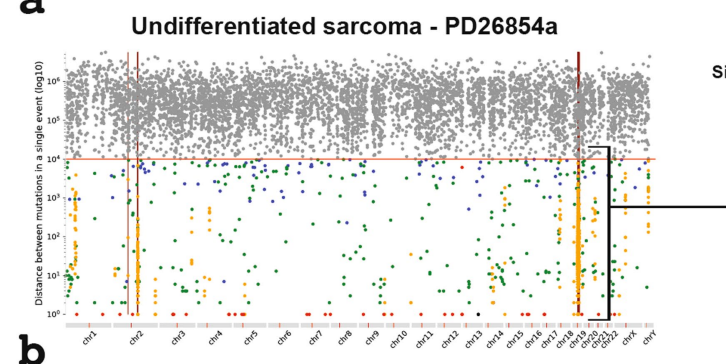

b

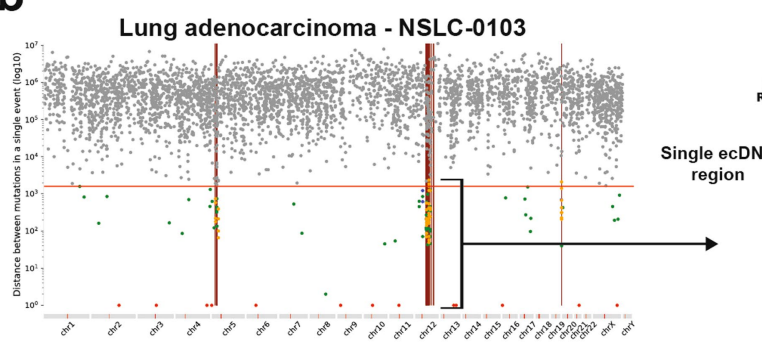

$\mathbf{C}$

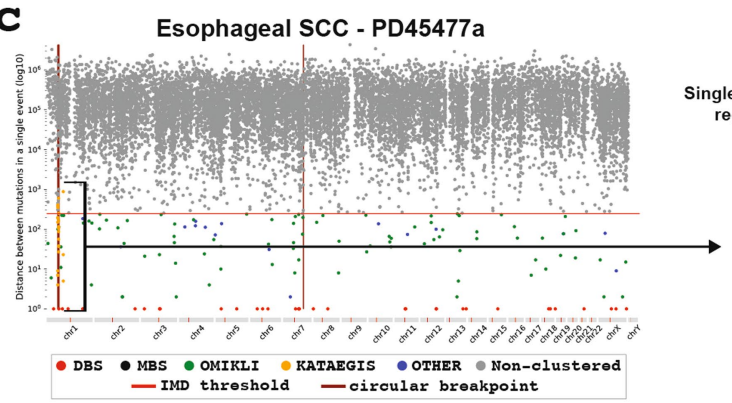

Extended Data Fig. 10 | Examples of kyklonas in three independent cohorts. a, A single undifferentiated sarcoma genome depicting the overlap of kataegis with ecDNA regions displayed as a rainfall (left) with a single zoomed in ecDNA represented using a circos plot (middle). The outer track of the circos plot represents the reference genome of the ecDNA with proximal known cancer driver genes. The middle track reflects a circular rainfall plot where each dot represents the IMD around a single mutation coloured based on the substitution change. The innermost track shows the average VAF for each kyklonic event. Right: Two smaller regions of the selected ecDNA including a single kyklonic event within ZNF536 region resulting in a plethora of missense

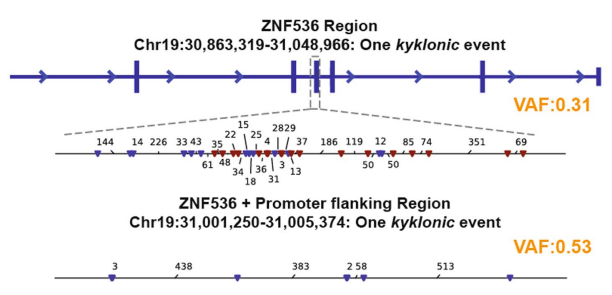

ZNF536 Region

BBC1D15 Region

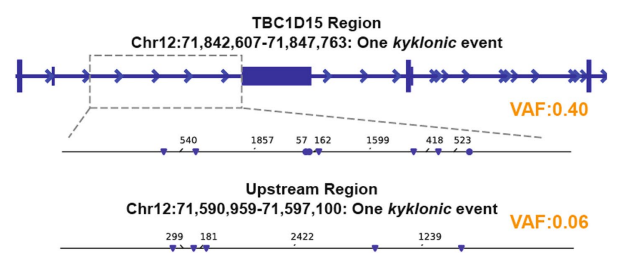

DAB1 Region

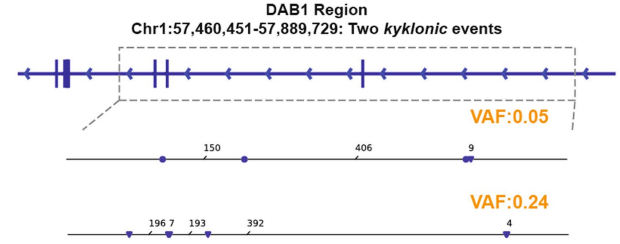

$\triangle \mathrm{C}>\mathrm{A} \bullet \mathrm{C}>\mathrm{G} \quad \nabla \mathrm{C}>\mathrm{T}$

$\nabla$ Missense and stop-gained mutations, and a single kyklonic event within a promoter flanking with the average VAFs per event (orange). b, A single lung adenocarcinoma genome depicting the overlap of kataegis with ecDNA regions (left) with a single zoomed in ecDNA containing TBC1D15 and two distinct kyklonic events represented using a circos plot (middle). Right: Two kyklonic events overlapping an upstream region and $T B C 1 D 15$. c, A single oesophageal squamous cell carcinoma genome depicting the overlap of kataegis with ecDNA regions (left) with a single zoomed in ecDNA containing $P R K A A 2$ and $D A B 1$ and three distinct kyklonic events (middle). Right: Two kyklonic events overlapping $D A B 1$. 


\section{Reporting Summary}

Nature Research wishes to improve the reproducibility of the work that we publish. This form provides structure for consistency and transparency in reporting. For further information on Nature Research policies, see our Editorial Policies and the Editorial Policy Checklist.

\section{Statistics}

For all statistical analyses, confirm that the following items are present in the figure legend, table legend, main text, or Methods section.

n/a Confirmed

\The exact sample size $(n)$ for each experimental group/condition, given as a discrete number and unit of measurement

$\bigotimes$ A statement on whether measurements were taken from distinct samples or whether the same sample was measured repeatedly

The statistical test(s) used AND whether they are one- or two-sided

Only common tests should be described solely by name; describe more complex techniques in the Methods section.

$\bigotimes$ A description of all covariates tested

$\bigotimes$ A description of any assumptions or corrections, such as tests of normality and adjustment for multiple comparisons

$\triangle$ A full description of the statistical parameters including central tendency (e.g. means) or other basic estimates (e.g. regression coefficient)

AND variation (e.g. standard deviation) or associated estimates of uncertainty (e.g. confidence intervals)

$\varnothing$ For null hypothesis testing, the test statistic (e.g. $F, t, r$ ) with confidence intervals, effect sizes, degrees of freedom and $P$ value noted Give $P$ values as exact values whenever suitable.

Х $\square$ For Bayesian analysis, information on the choice of priors and Markov chain Monte Carlo settings

Х $\square$ For hierarchical and complex designs, identification of the appropriate level for tests and full reporting of outcomes

$\bigotimes$ Estimates of effect sizes (e.g. Cohen's $d$, Pearson's $r$ ), indicating how they were calculated

Our web collection on statistics for biologists contains articles on many of the points above.

\section{Software and code}

Policy information about availability of computer code

Data collection No data were generated specifically for this study. All data were and can be downloaded from the appropriate links, repositories, and references. Specifically, for the discovery cohort, all data and metadata were obtained from the official PCAWG release: https://dcc.icgc.org/ releases/PCAWG. All data and metadata for TCGA samples were obtained from GDC: https://gdc.cancer.gov/. Genomics data for clonally expanded cell lines were downloaded from European Genome-phenome Archive: EGAD00001004201, EGAD00001004203, and EGAD00001004583. For the three validation cohorts, datasets were downloaded as submitted by the original publications and genomics data were downloaded from their respective repositories: EGAD00001004162 for 61 undifferentiated sarcomas (European Genome-phenome Archive), EGAD00001006868 for 187 high-confidence esophageal squamous cell carcinomas (European Genome-phenome Archive), and phs001697.v1.p1 for 280 lung adenocarcinomas (dbGaP). Somatic mutations and metadata for the MSK-IMPACT Clinical Sequencing Cohort composed of 10,000 clinical cases were downloaded from cBioPortal: https://www.cbioportal.org/study/summary?id=msk_impact_2017.

Data analysis The SigProfiler compendium of tools are developed as Python packages and are freely available for installation through PyPI or directly through GitHub (https://github.com/AlexandrovLab/). For all tools, each package is fully functional, free, and open sourced distributed under the permissive 2-Clause BSD License and are accompanied by extensive documentation: (i) SigProfilerMatrixGenerator (version 1.2.0; https:// github.com/AlexandrovLab/SigProfilerMatrixGenerator); (ii) SigProfilerSimulator: (version 1.0.2; https://github.com/AlexandrovLab/ SigProfilerSimulator); (iii) SigProfilerExtractor: (version 1.1.0; https://github.com/AlexandrovLab/SigProfilerExtractor). Each SigProfiler tool also has an R wrapper available for installation through the GitHub repositories. AmpliconArchitect (version 1.2) is also freely available and can downloaded from https://github.com/virajbdeshpande/AmpliconArchitect. The core computational pipelines used by the PCAWG Consortium for alignment, quality control and variant calling are available to the public at https://dockstore.org/search?search=pcawg under the GNU General Public License v.3.0, which allows for reuse and distribution. 
Policy information about availability of data

All manuscripts must include a data availability statement. This statement should provide the following information, where applicable:

- Accession codes, unique identifiers, or web links for publicly available datasets

- A list of figures that have associated raw data

- A description of any restrictions on data availability

No data were generated specifically for this study. All data were and can be downloaded from the appropriate links, repositories, and references. Specifically, for the discovery cohort, all data and metadata were obtained from the official PCAWG release: https://dcc.icgc.org/releases/PCAWG. All data and metadata for TCGA samples were obtained from GDC: https://gdc.cancer.gov/. Genomics data for clonally expanded cell lines were downloaded from European Genome-phenome Archive: EGAD00001004201, EGAD00001004203, and EGAD00001004583. For the three validation cohorts, datasets were downloaded as submitted by the original publications and genomics data were downloaded from their respective repositories: EGAD00001004162 for 61 undifferentiated sarcomas (European Genomephenome Archive), EGAD00001006868 for 187 high-confidence esophageal squamous cell carcinomas (European Genome-phenome Archive), and phs001697.v1.p1 for 280 lung adenocarcinomas (dbGaP). Somatic mutations and metadata for the MSK-IMPACT Clinical Sequencing Cohort composed of 10,000 clinical cases were downloaded from cBioPortal: https://www.cbioportal.org/study/summary?id=msk_impact_2017.

\section{Field-specific reporting}

Please select the one below that is the best fit for your research. If you are not sure, read the appropriate sections before making your selection. $\bigotimes$ Life sciences $\quad \square$ Behavioural \& social sciences $\quad \square$ Ecological, evolutionary \& environmental sciences

For a reference copy of the document with all sections, see nature.com/documents/nr-reporting-summary-flat.pdf

\section{Life sciences study design}

All studies must disclose on these points even when the disclosure is negative.

Sample size No sample size calculation was performed as no data were being generated. Rather, all publicly available samples were utilized in our analysis and, for each comparison sufficient numbers were determined based on a FDR-corrected statistically significant p-value and magnitude of effect size. Overall, the study utilized the complete set of 2,583 white-listed whole-genome sequenced samples from PCAWG along with their corresponding list of consensus driver events. Samples were taken as provided by the PCAWG consortium. Extrachromosomal-DNA (ecDNA) can be unambiguously assigned to 1,291 of these samples. Validation cohorts included 61 sarcomas, 280 lung cancers, and 186 esophageal squamous cell carcinomas.

Data exclusions No samples were exclude in the discovery PCAWG cohort. All PCAWG cancer types with more than 10 samples are presented within the main figures while cancer types with less than 10 samples are included in Extended Data Figures. In the validation esophageal cohort, only highconfidence esophageal squamous cell carcinomas were used as annotated in the submission to the data repository. No samples were excluded from the sarcoma and lung cancer validation cohorts.

Replication Replication of genomics analyses encompassed three independent cohorts and a total of 527 additional whole-genome sequenced samples, including: 61 sarcomas, 280 lung cancers, and 186 esophageal squamous cell carcinomas. The results from the genomics analyses of PCAWG were replicated three times -- one per each validation cohort. Additionally, the MSK-IMPACT Clinical Sequencing Cohort composed of 10,000 clinical cases was used for clinical validation. The results from the clinical analysis of TCGA clustered cancer genes was replicated one time -- in the MSK-IMPACT Clinical Sequencing Cohort.

Randomization There was no sample randomization in this study. Rather, the performed statistical analyses controlled for most known confounders Specifically, in the clinical association analyses, we corrected for age of diagnosis (where available), tumor mutational burden, and cancer type. For most statistical comparisons between clustered mutations, a correction was performed based on observed behavior of nonclustered mutations.

Blinding Detection of clustered mutations was performed independently and in a blinded manner in regard to driver mutations, overall survival, and identification of extrachromosomal-DNA (ecDNA).

\section{Reporting for specific materials, systems and methods}

We require information from authors about some types of materials, experimental systems and methods used in many studies. Here, indicate whether each material, system or method listed is relevant to your study. If you are not sure if a list item applies to your research, read the appropriate section before selecting a response. 
Materials \& experimental systems Methods

\begin{tabular}{l|l}
\hline n/a & Involved in the study \\
$\searrow$ & $\square$ Antibodies \\
$\searrow$ & $\square$ Eukaryotic cell lines \\
$\searrow$ & $\square$ Palaeontology and archaeology \\
$\searrow$ & $\square$ Animals and other organisms \\
$\searrow$ & $\square$ Human research participants \\
$\searrow$ & $\square$ Clinical data \\
$\searrow$ & $\square$ Dual use research of concern
\end{tabular}

$\mathrm{n} / \mathrm{a}$ Involved in the study

$\bigotimes \square$ ChIP-seq

$\bigotimes \square$ Flow cytometry

$\bigotimes \mid \square$ MRI-based neuroimaging

$\bigotimes \mid \square$ Dual use research of concern 